NBS

PUBLICATIONS

\title{
Cooperative Research Opportunities at NBS
}

NATIONAL BUREAU OF STANDARDS • U. S. DEPARTMENT OF COMMERCE

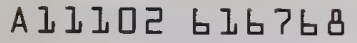

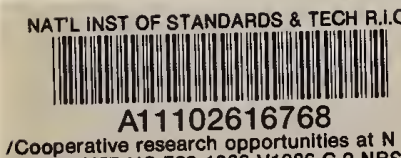

QC100.U57 NO.723 1986 V1986 C.2 NBS-PUB

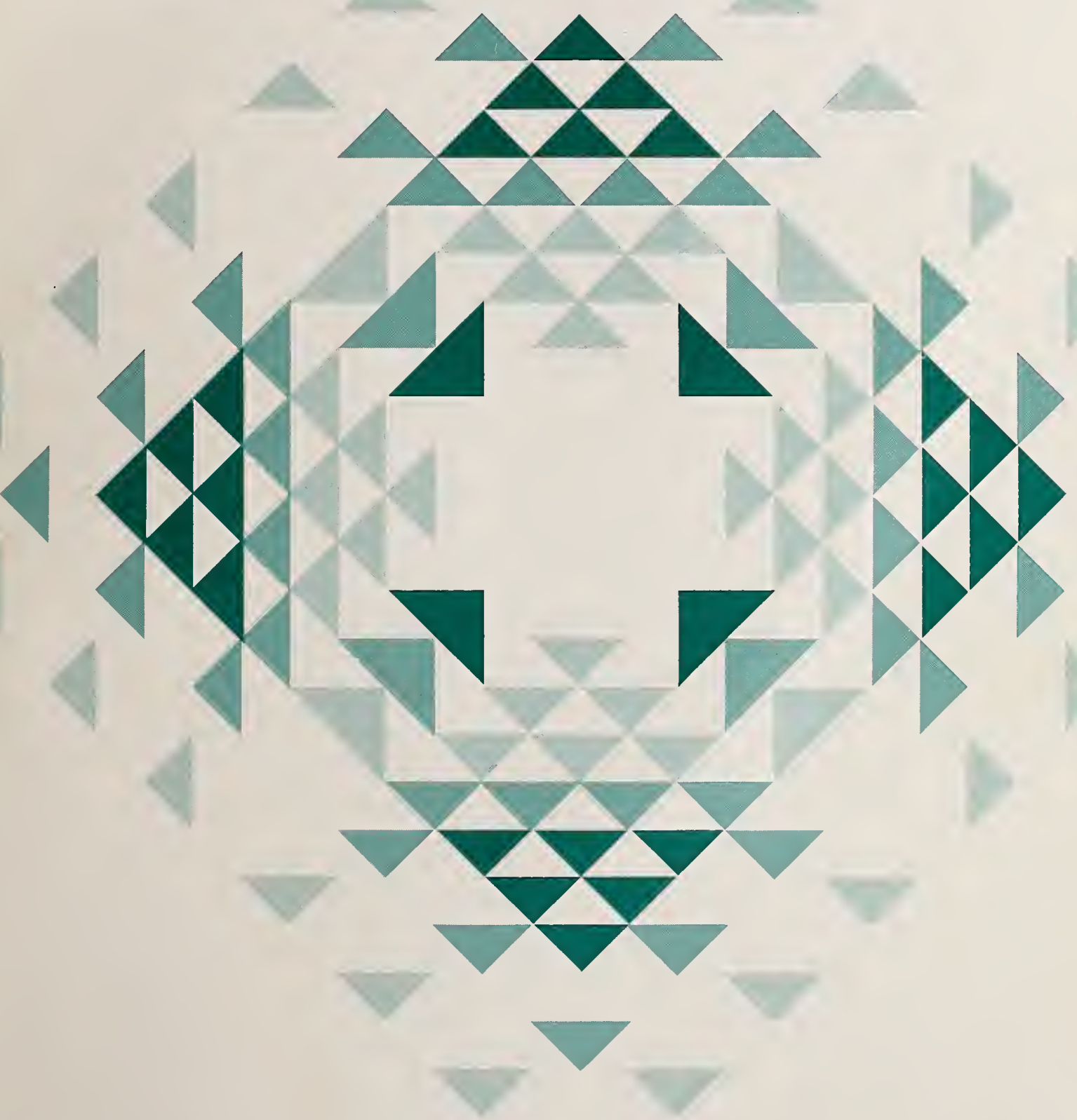

$Q C$

100

.457

No.723

1986

C. 2 

Message from the Director

Research Opportunities

Analytical Chemistry

Applied Mathematics

Basic Standards

Building Technology 19

Ceramics 22

Chemical Engineering

Chemical Physics 29

Computer Sciences and Technology 31

Electronics and Electrical Engineering 33

Fire Research 37

Fracture and Deformation 38

Manufacturing Engineering 39

Metallurgy 40

Neutron Scattering and Diffraction 43

Nondestructive Evaluation 44

Polymers 45

Product Standards 48

Radiation Research 49

Standards Reference Data 52 


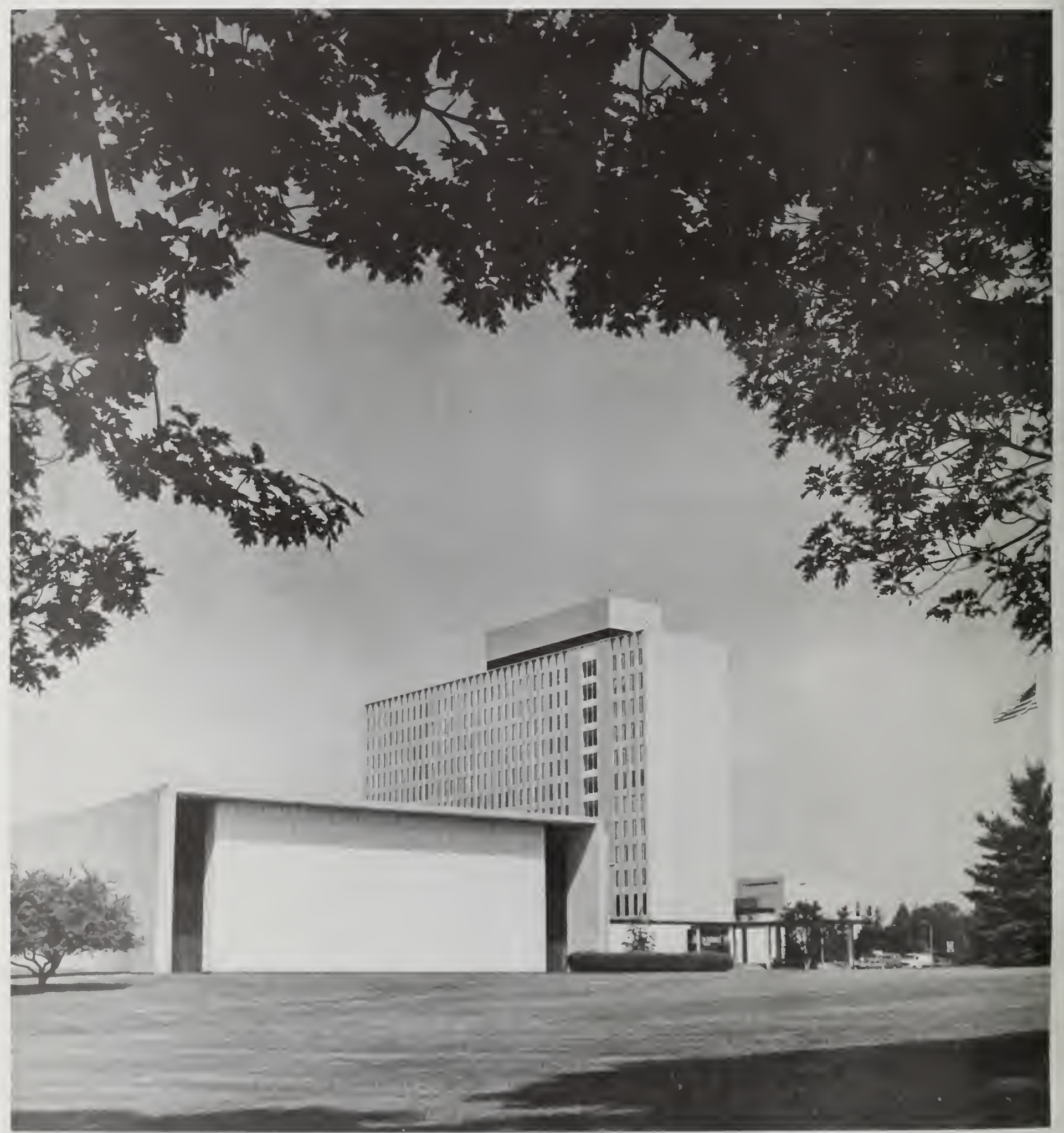




\section{Message from the Director}

Our primary goal as the nation's physical sciences and engineering measurement laboratory is to serve industry and science-to make sure our research is helping the nation solve its measurement problems. By doing so, we help industry exploit the nation's science and technology in its quest to compete successfully in the international marketplace.

At the National Bureau of Standards, we found long ago that one of the most effective ways to accomplish this is through cooperative research. Each year more than 900 people from industry, universities, and other government agencies work side-byside with NBS scientists and engineers on a wide range of research projects including automated manufacturing, biotechnology, computer networking, and mechanical failure of materials.

Because I firmly believe we will best meet today's economic and technological challenges by working together, we are increasing the opportunities for cooperative research here at the National Bureau of Standards. Let me mention just briefly the advantages, as I see them, of collaborative research to both NBS and the cooperating organization.

Researchers from industry, universities, and other government agencies get an opportunity to work with NBS specialists, many of whom are renowned experts in their fields, and to use the Bureau's premier research and testing facilities. They take newly developed technology back to their organizations to be incorporated immediately into new ideas, new processes, and new products. NBS researchers, in turn, learn firsthand the views and needs of Bureau clients and, as a result, we are better able to direct our research to meet these needs.

One of our longest running cooperative projects has been a remarkably successful collaboration with the American Dental Association. Started in 1928, the alliance has resulted in the panoramic $x$-ray machine, composite restorative materials, and the high-speed turbine handpiece, for example, as well as major contributions to dozens of standards for dental materials and hundreds of scientific papers.

The scope, duration, and structure of our cooperative projects are tailored to the problem to be solved. They can last for a few weeks or a few years and involve one, several, or even hundreds of organizations. Our goal is to solve the problem at hand quickly, efficiently, and effectively.

I invite you to review the research opportunities described in this brochure, and then to call us to discuss the possibilities for collaborative research in an area that is of concern to both of us. By working together, we can facilitate technology transfer, help improve industrial productivity, and strengthen this nation's ability to compete in international markets.
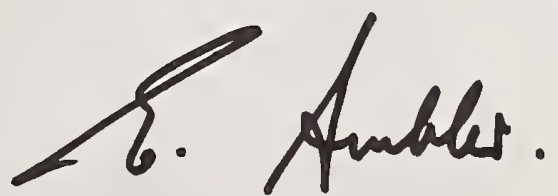

Ernest Ambler

Director 


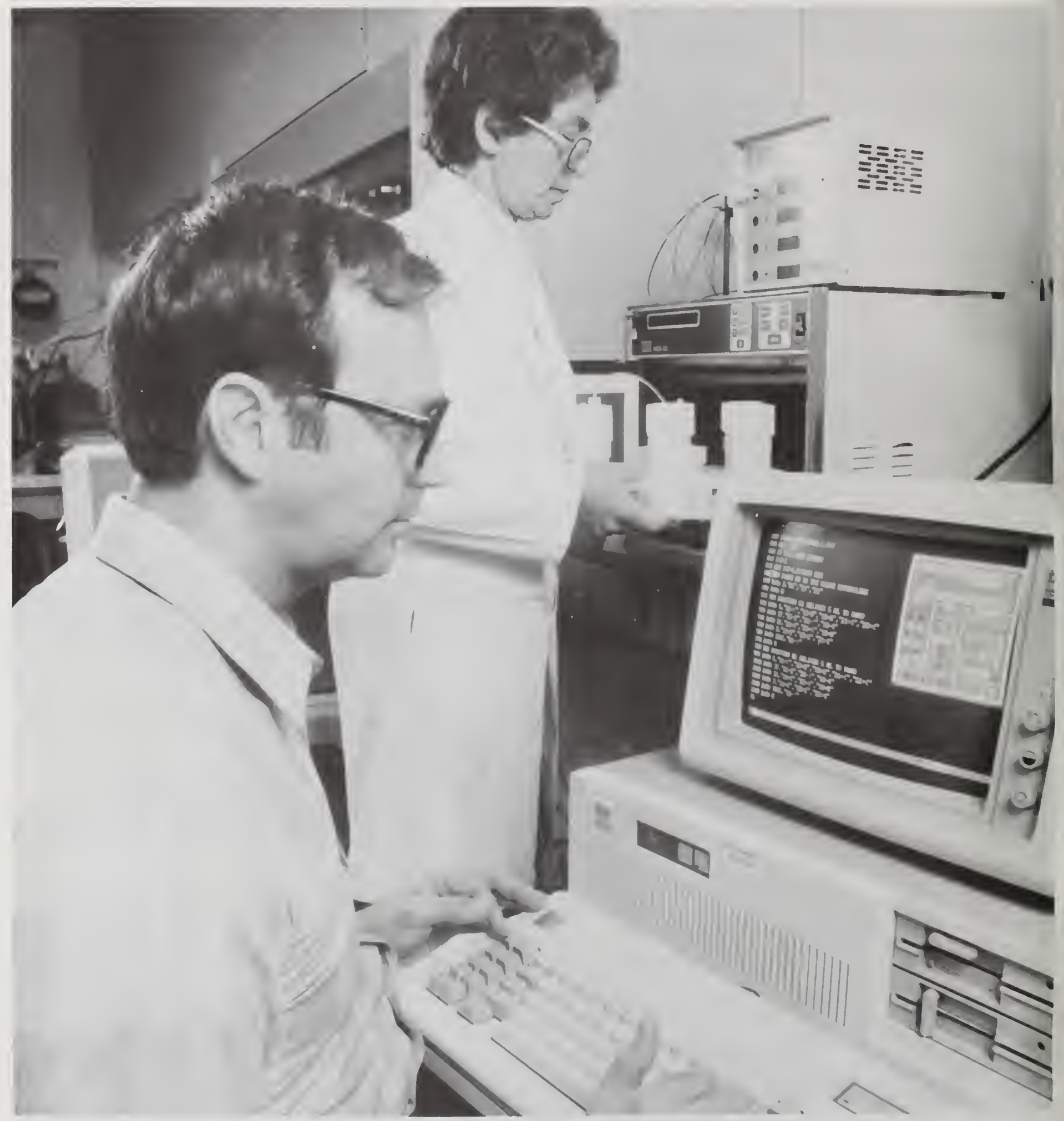

NBS chemist Howard Kingston and Lois Jassie, a chemist from CEM Corporation, have designed microwave techniques that can be used to dissolve chemical samples more safely and efficiently than traditional methods. 


\section{Cooperative Research at NBS}

Numerous opportunities exist for scientists and engineers from industrial, professional, trade, and other organizations to collaborate in research at the National Bureau of Standards on projects of mutual interest. To accomplish its objective of supplying the measurement foundation for industry, science, and technology, NBS conducts research in many areas, including advanced ceramics, automated manufacturing, optoelectronics, and biotechnology.

Operated on an annual budget of about $\$ 210$ million, the Bureau employs a highly skilled staff of some 2,700 at its headquarters in Gaithersburg, Md., and its site in Boulder, Colo. NBS also has extensive research and testing facilities that are available for use in cooperative research, such as a 20-megawatt research reactor, an electron accelerator, a synchrotron ultraviolet radiation facility, a supercomputer, a large-scale structures testing facility, a metals-processing laboratory, a fire research facility, and a computer network protocol testing and evaluation laboratory.

NBS will work with a company, trade association, university, or other government agency to solve problems of mutual concern. One of the most popular ways NBS research and facilities are made accessible to U.S. industry is through the Bureau's Industrial Research Associate Program. Under this program, companies, trade associations, and technical societies pay their scientists and engineers to work in NBS laboratories with NBS researchers on a specific project.

In another type of cooperative venture, companies subscribe to a consortium that provides resources to have NBS solve a specific measurement problem facing the industry. This arrangement has advantages when a complex problem confronts a particular industry and the research is too costly or not possible for individual companies to undertake.

Companies also loan or donate equipment or software to the Bureau when NBS researchers are working on a project that could ultimately improve their products or the way they do business. In such situations, these firms actively consult or advise NBS researchers on the needs and concerns of the industry. In other cases, NBS makes its facilities available to companies for proprietary research on a cost-recovery basis, when equal or superior facilities are not otherwise available.
Described below are a few brief examples of different types of cooperative projects and the results they have produced so far

\section{Manufacturing Research Facility}

The NBS Automated Manufacturing Research Facility (AMRF), a research center for studying fundamental questions affecting factory automation, is one of the largest cooperative programs at the Bureau. Since 1982, 35 companies-large and small-have paid more than 50 of their researchers to work in the AMRF, and about $\$ 4.6$ million worth of equipment and software has been loaned or donated to NBS by industry for this project. The Navy Manufacturing Technology Program is a major partner in the facility, and the Air Force Intelligent Task Automation Project and other government agencies also have sponsored specific parts of the AMRF effort. In addition, a number of universities have contributed to the project by loaning guest researchers.

Together these researchers are experimenting with methods for getting robots, computers, and machine tools from different manufacturers to communicate and work together in an integrated system through standardized interfaces. Their other primary goal is to find a means for controlling quality in a fully automated factory environment by improving the measurement process.

All of this will give companies, in particular small firms with less than 50 employees, the flexibility to buy equipment from different manufacturers at different times with the assurance that the machines they buy can be made to work together without expensive, custom-designed interfaces. Concepts developed at the AMRF are now being adopted by hardware manufacturers and the U.S. Navy.

According to a study by the Management Collaborative Group of Chapel Hill, N.C., the contribution of the AMRF to the increased use of numerical-control machine tools in U.S. industry will produce a savings to the metalworking industry of between $\$ 250$ and $\$ 760$ million per year by 1990 .

\section{Chemical Engineering Consortia}

NBS recently completed a 4-year research project on the properties of supercritical fluids which was sponsored by a consortium of 14 private companies and 
the Department of Energy. The goal of this research was to develop accurate predictive models for supercritical fluid mixtures and accurate experimental data that can be used to test and further develop the predictive models. Among the results of this effort is a state-of-the-art computer code to be used in the custody transfer of supercritical fluid mixtures.

Meetings of the consortium participants were held twice a year where research results were disseminated and direction of the project discussed. This effort was judg ed so successful by the participants that two new consortia were formed from it.

NBS researchers are working on two other consortium-sponsored projects in chemical engineering. One consortium, which is studying the measurement problems that result when a meter is installed too close to a pipe elbow or valve, has 10 firms and the Department of Energy as members. The other consortium has a roster that includes nine companies and the National Aeronautics and Space Administration, all of which are interested in im. proving the performance of vortex-shedding flow meters.

\section{Collaborative Research in Microwave Technology}

To refine techniques for rapidly dissolving samples in closed vessels within a microwave chamber, CEM Corporation, a producer of microwave equipment for research purposes, sent one of its chemists to NBS to work as an industrial research associate with Bureau researchers. As a result of this cooperative project, microwave technology can now be used to dissolve samples-everything from biological specimens such as human bone and bovine livers to botanical, geological, metallic, and glassy samples-more safely and efficiently than traditional methods.

By combining the microwave technique's ability to heat materials quickly with the superior dissolving properties of closed containers under pressure, the NBS/CEM research team has designed a method for breaking down samples that takes less than 10 minutes. Traditionally, using hot plates and open-vented beakers, scientists could expect dissolving times of anywhere from 4 hours to 4 days, depending on the complexity of the sample.
The researchers have produced a database of information that will allow industrial chemists to tailor their own microwave devices for desired results. They are also helping others apply their techniques: So far they have given information and advice to more than 250 researchers from companies, universities, and research laboratories.

\section{Biotechnology Center}

In a first-of-its-kind agreement for NBS, the Bureau, The University of Maryland, and Montgomery County, Md., are establishing a Center for Advanced Research in Biotechnology (CARB) to provide a forum for collaborative research among academic, government, and industrial scientists. When the new CARB building is completed in December 1987, it is expected to house state-of-the-art facilities and 100 researchers from NBS, The University of Maryland, industry, and other government agencies and universities.

The goal of the center is to radically reduce the time and effort required to determine the atomic structure of proteins and to model and predict their properties. This research will help build the foundation for the rapidly emerging fields of protein engineering and rational drug design.

\section{Phase Diagram Center}

ASM International has raised $\$ 4$ million as industry's portion of a cooperative ASM/NBS program that will give scientists and engineers more reliable data, in the form of alloy phase diagrams, for designing new and better metal alloys and products in which metal alloys are used. Fifty companies, organizations, institutes and agencies, and hundreds of individuals made contributions to ASM.

Researchers from NBS and industry work in the Alloy Phase Diagram Center, which is located at NBS. ASM and NBS also support individual data evaluation programs in other laboratories. The critically evaluated phase diagrams, which are graphic representations of what happens when two or more elements are com. bined at various temperatures and pressures, are published by ASM as part of the collaborative effort. Data from the ASM/NBS program are expected to save U.S. firms hundreds of millions of dollars. 


\section{Computer Networking Workshops}

For the past several years, NBS has been working with industry and standards groups to make it easier for those using computer systems or equipment to communicate with each other through computer networks regardless of who manufactures the individual pieces. NBS has cooperated with the standards groups to develop the needed standards and with industry to implement and test the standards in commercial products for the office and factory.

As part of this project, NBS sponsors a series of workshops for vendors and users to discuss the implementation of the Open Systems Interconnection (OSI) Reference Model. To date, over 200 companies, including most major computer manufacturers, communications carriers, semiconductor companies, factory control system companies, and word processing manufacturers, as well as government agencies have participated in the workshops.

Two successful demonstrations-one at the 1984 National Computer Conference, the other at AUTOFACT ' 85 -of standards for the OSI reference model have resulted. These cooperative activities have now led to the use of new computer network protocols by several hundred companies for manufacturing and office automation.

\section{How to Participate}

These examples illustrate the range of cooperative programs that it is possible to establish-from a small, very focused project with limited resources to those in which numerous organizations are contributing to research that will affect an entire industry. If you are interested in working with NBS on a project, large or small, write or call one of the offices listed below or one of the contacts given in the Research Opportunities section of this brochure.

- Industrial Research Associate Program Under this program, which NBS has run since the 1920's, scientists and engineers from industry, trade associations, technical societies, and other organizations work side-by-side with NBS researchers. With their salaries paid by their employers, the associates work at NBS, usually for 1 to 3 years, on a wide range of research includ ing robotics, computer science, advanced ceramics, and nondestructive analysis. For further information, contact the NBS Office of Research and Technology Applications, 301/975-3087.

- Proprietary Facilities While most NBS facilities are available for cooperative research, the Bureau recently opened up selected facilities for proprietary research. NBS makes these facilities available under certain conditions to support U.S. industry in its development of new competitive products and processes. For additional information, contact the NBS Program Office, 301/975-2657.

- Other Arrangements

To inquire about other types of cooperative research programs with NBS, such as a consortium or research center, contact the appropriate institute or laboratory.

$\square$ Institute for Materials Science and Engineering, 301/975-5658: Provides standards, measurement methods, data, and quantitative understanding to aid in improving the processing and performance of metals, polymers, ceramics, composites, and glasses.

$\square$ National Measurement Laboratory, 301/975-4500: Provides the nation with state-of-the-art measurement services in thermodynamics, transport properties, chemical kinetics, surface science, molecular spectroscopy, and chemical analysis. Conducts research to improve realization of basic physical quantities.

$\square$ Institute for Computer Sciences and Technology, 301/975-2819: Provides the standards, specifications, measurement and test methods, and technical guidance needed by government and industry to make better use of computer hardware, software, networks, and security.

$\square$ National Engineering Laboratory, 301/975-2301: Conducts research in engineering and applied science. Studies problems in electronics, manufacturing automation, chemical engineering, the behavior (and prevention) of fires, and the design and construction of buildings. 


\section{Research Opportunities}

NBS welcomes collaboration with scientists and engineers from industry, universities, and other government agencies on most of its projects. To give those interested in cooperative research an idea of the wide range of NBS programs, a sampling of specific Bureau research projects is described on the following pages.
To discuss the possibility of setting up a cooperative research project in one of these areas, write or call the contact listed. (Unless otherwise specified, all contacts are at the National Bureau of Standards,

Gaithersburg, MD 20899.) For general information on cooperative research at NBS, write or call one of the offices listed on page 7 .

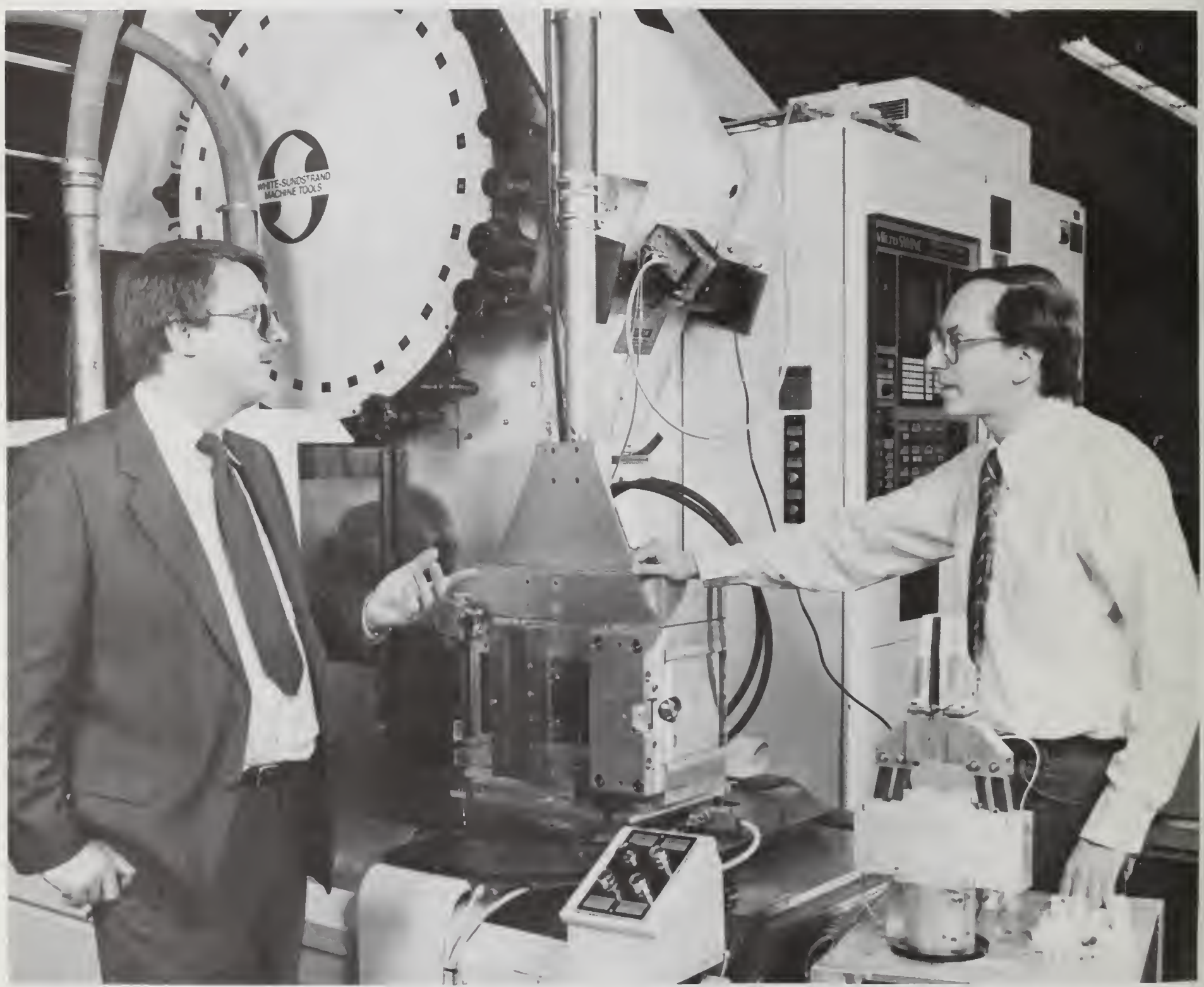

At the horizontal workstation in the NBS Automated Manufacturing Research Facility, physicists Christopher Stevenson (left) of White-Sundstrand and Howard Harary of NBS discuss the next generation of machine tools. 


\section{Analytical Chemistry}

Atomic Atomic spectroscopy methods are probSpectroscopy ably the most widely used analytical techniques in industry today. Considerable research is required, however, to keep up with the changing needs of industry. NBS research in atomic spectrometry is focused on several different studies. For example, researchers are working to improve the analytical capabilities of the direct current plasma (DCP) and the inductively coupled plasma (ICP), as well as experimenting with the glow discharge as an atom reservoir. Additional research concerns the use of spark sampling for the direct analysis of solids, the continued development of laserenhanced ionization (LEI) in flames, and the evaluation of various types of coupled chromatographic-spectrometric systems for improving the accuracy of spectroscopic measurements.

NBS scientists also are developing a series of neutral density filters that can be issued as Standard Reference Materials for verifying the accuracy of the transmittance and absorbance scales of ultraviolet, visible, and near infrared absorption spectrophotometers.

Contact: Robert L. Watters, B222 Chemistry Bldg., 301/975-4122.

Analytical Mass Analytical mass spectrometry has played Spectrometry a key role in assisting industries, such as the semiconductor industry, which require accurate measurements of trace elements in their raw materials, products, and product containers. Bureau research in inorganic mass spectrometry is concerned with developing analytical capabilities for the highly accurate determination of trace inorganics by stable isotope dilution, the highly precise meas urement of isotopic compositions, and the highly accurate measurement of absolute isotopic compositions for the redetermination of atomic weights. This research involves development of instrumentation in spark source, thermal source, inductively coupled plasma source, and laser source mass spectrometry and research in chemical separations at the trace level using ion chromatography and other techniques.

Contact: John D. Fassett, A21 Physics Bldg., 301/975-4109.
Recently, electroanalytical chemistry has had a key role in the development of methods and materials for environmental and clinical determinations, in particular, $\mathrm{pH}$ and conductance. While Bureau research interests and activities include most areas of electroanalytical and classical chemistry, current emphasis is on electrochemical detector development, voltammetric and ion chromatographic measurement methods, high-purity assay by coulometric titration, potentiometric measurements in biological fluids, and fundamental studies and measurements in $\mathrm{pH}$ and aqueous electrolytic conductance. NBS scientists are giving special attention to the development of novel electroanalytical instrumentation, including computer interfacing. For all these projects, instrumentation is available for accurate $\mathrm{pH}$, potentiometric, coulometric, conductometric, voltammetric, and ionchromatographic measurements.

Contact: William F. Koch, A225

Chemistry Bldg., 301/975-4132.

More than 50 billion chemical analyses are performed annually in the United States. The Bureau helps to ensure the accuracy of these analyses by developing new analytical procedures and improving the reliability and accuracy of present methods. One technique under study is activation analysis, which is a highly sensitive, nondestructive, analytical technique not normally available in most industrial laboratories. In this research, methods of nuclear analysis are investigated utilizing the 20-MW NBS research reactor. Research is performed in all areas of the technique including the capabilities of cold-neutron activation, the use of sample selfconsistent (monitor) activation, the determination of new mathematical procedures for the resolution of gamma spectra, the development of prompt gamma activation techniques, and the use of chargedparticle activation techniques.

Contact: Ronald F. Fleming, B108 Reactor Bldg., 301/975-6279.

\section{Inorganic Electroanalytical Research}

\section{Activation Analysis}


Liquid Liquid chromatography is one of the Chromatography/ most widely used analytical techniques in Electrochemistry industrial laboratories. While this technique has achieved widespread application, its uses can be broadened even further by improvements in detection methods. The focus of NBS research is the development of novel approaches for the electrochemical detection of organic analytes separated by liquid chromatography (LC). To apply this technique most effectively, it is essential to investigate the electrode reaction mechanisms of the compounds to be detected using techniques such as cyclic and reverse-pulse voltammetry, coulometry, and LC/ultraviolet spectroscopy. Ongoing work focuses on the use of differential pulse and dual-electrode detection, as well as the development of single and array microelectrode detectors. Researchers also are developing new liquid chromatographic separations employing specific chemical interactions including chelation, ion-pairing, charge transfer complexation and acid-base equilibria.

Contact: William A. MacCrehan, A113 Chemistry Bldg., 301/975-3122.

Bioanalytical Biosensors are the newest generation of Sensors analytical devices having the potential for widespread use in biomedical and industrial monitoring applications. These devices will incorporate the latest advances in biotechnology to provide sensors of high specificity and sensitivity. Biologically derived substances have important application as components of rapid-sensing devices because of their binding specificity, the strength of their interactions, and their potential for use in a wide variety of amplification schemes. Immunological, enzymatic, and receptorligand interactions are being explored as the basis for analytical devices.

NBS researchers are employing a variety of optical and electrochemical techniques for detection. Detection can be based on such diverse changes as size and rotational mobility of analytes or binding agents upon interaction, or can be the result of enzymatic activity which occurs due to analyte binding, causing enhanced fluorescence or electrochemical signal. Amplification can be achieved with release to liposome-encapsulated molecules, multi-turnover enzyme reactions, ion-exchange polymer-modified electrodes, and so forth. The combination of high specificity for analytes with efficient amplification provides the potential for extremely sensitive devices.

Contact: Richard A. Durst, A113 Chemistry Bldg., 301/975-3118.

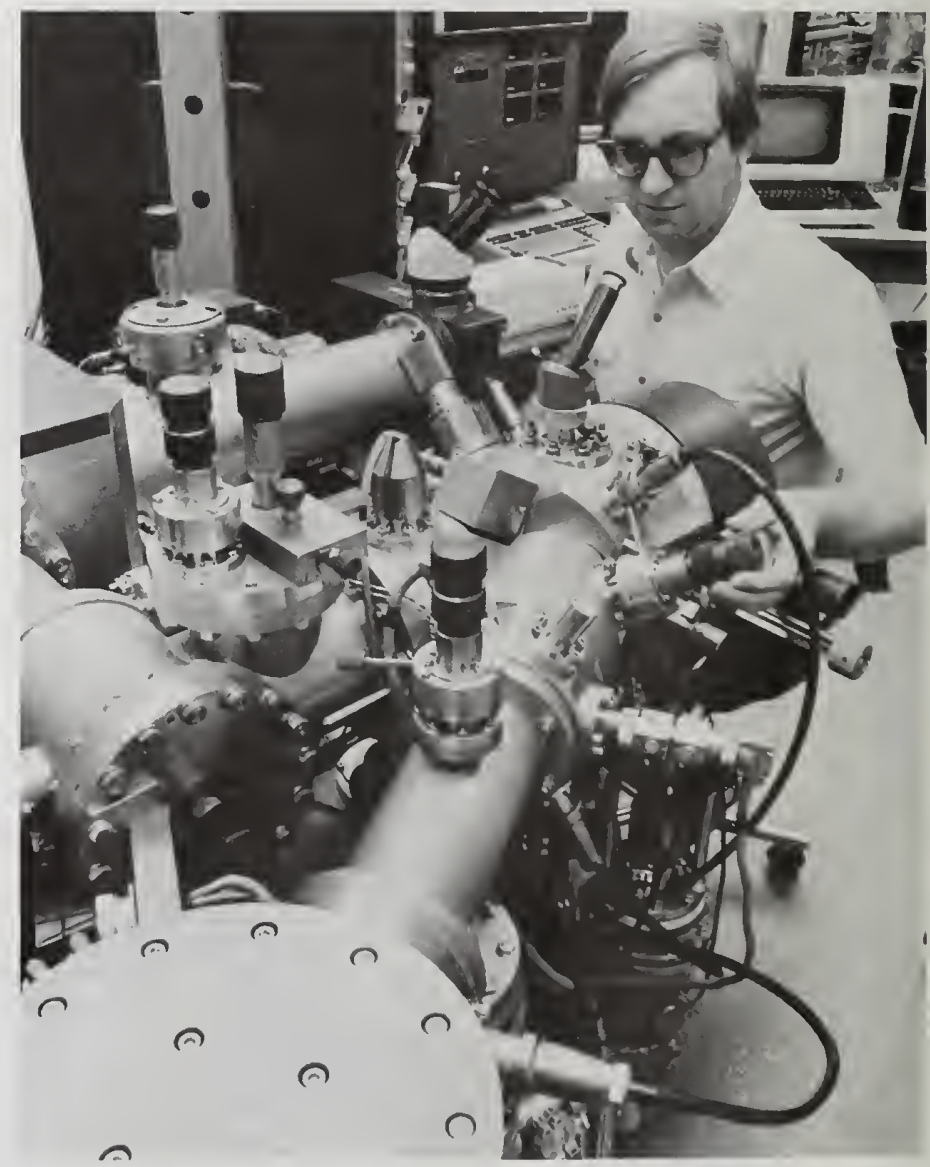

Using a secondary ion mass spectrometry instrument, metallurgist Dale Newbury is able to map the distribution of elements both on and below a sample's surface. 
Supercritical Supercritical fluid chromatography (SFC)

Fluid in both capillary and packed columns

Chromatography has recently received renewed attention because the process offers several advantages over liquid and gas chromatography for high-efficiency separations of nonvolatile molecules. Potential NBS research in this area would focus on the investigation of the variables that influence the retention, selectivity, and efficiency of SFC systems and on the development of SFC as a useful analytical technique to provide high-efficiency separations of complex mixtures. A packed-column SFC is available and a capillary SFC is being procured to make state-of-the-art measurements. Auxiliary spectroscopic detectors have and are being developed to complement this SFC instrumentation.

Contact: Stephen N. Chesler, 301/9753102 or Stephen A. Wise, 301/975-3112, A113 Chemistry Bldg.

Liquid Liquid chromatography (LC) on chemiChromatographic cally bonded stationary phases (e.g., $\mathrm{C}_{18}$, Stationary $\mathrm{C}_{8}, \mathrm{NH}_{2}$, and $\mathrm{NO}_{2}$ ) offers unique capabili-

Phases: ties for the separation of isomeric

Retention compounds or compound classes.

Mechanisms and However, significant differences in retenCharacterization tion and selectivity characteristics, such as relative retention, are observed among commercial phases produced by different manufacturers and even within different batches prepared by the same manufacturer.

One area of NBS research has been directed toward understanding which factors influence LC retention on these various stationary phases to predict, improve, and control the separation selectivity. In reversed-phase $\mathrm{LC}$ on $\mathrm{C}_{18}$ phases, polycyclic aromatic hydrocarbons have been used as model nonpolar solutes to study retention mechanisms and selectivity. Various physical parameters of these solutes, including size, shape, aqueous solubility, and partition coefficients; characteristics of the bonded phase such as phase type, alkyl chain length, and ligand surface density; and mobile phase characteristics have been studied to determine their influence on retention and selectivity in LC. Investigations have focused on both the physical and chromatographic characterization of these stationary phases.

A variety of methods are available at NBS for physical characterization of these materials, including carbon analysis, gas adsorption surface area techniques, infrared and nuclear magnetic resonance spectroscopy, and small-angle neutron scattering techniques. Currently, Bureau scientists are attempting to develop chromatographic test mixtures to evaluate LC stationary phase characteristics and chromatographic performance. Such "performance" standards would allow the direct comparison of the various LC phases produced by different manufacturers and provide insight into the development of improved stationary phases for specific separations.

Contact: Stephen A. Wise, A113 Chemistry Bldg., 301/975-3112.

Multidimensional (dual column) and very high-resolution ( $>100,000$ plates) gas chromatographic methods offer the analyst an opportunity to perform difficult separations to facilitate species-specific quantitation. These high-resolution techniques are being investigated at NBS to develop highly specific analytical procedures for the quantitation of $\mathrm{ng} / \mathrm{g}$ organic constituents contained in complex environmental and clinical matrices

Research in this area focuses on the optimization of multidimensional gas chromatographic experiments in which two capillary columns, each possessing individual and different retention mechanisms, are serially connected by a pneumatic switch. In addition, studies center on the use and development of tailored, very high-resolution capillary columns. An independently controllable, dual-oven gas chromatograph is available for multidimensional research. Additional facilities and equipment include state-ofthe-art sample preparation and gas chromatography laboratories, 12 capillary gas chromatographs with multiple specific and non-specific detectors, and a large laboratory information management system (LIMS).

Contact: Stephen N. Chesler, A113 Chemistry Bldg., 301/975-3102.
Multidimensional and Very HighResolution Gas Chromatography 
Microbeam Interpreting the relationship between the Compositional physical and chemical microstructure of Mapping materials is important in understanding their macroscopic behavior and in extending their in-service performance. Conventional microbeam techniques for elemental/molecular compositional analysis on the micrometer scale, such as the electron microprobe and ion microscope, have been restricted to quantitative analysis at individual locations. Mapping of the distribution of constituents has been possible only at the qualitative or semiquantitative level. However, recent NBS research developments have led to the production of the first true quantitative compositional maps. Quantitative compositional mapping with the electron microprobe has been demonstrated down to levels of 0.1 weight percent, while quantitative isotope ratio measurement in images has been demonstrated with the ion microscope.

Current research activities include extension of compositional mapping to analytical electron microscopy, laser Raman microanalysis, and laser microprobe mass analysis.

Potential projects in this field include applying the compositional mapping instruments to materials characterization problems, developing new techniques for compositional mapping on other microanalysis instruments, and investigating basic topics in quantitative analysis with microbeam instrumentation.

The equipment available for this activity includes a Cameca electron microprobe, a JEOL analytical scanning electron microscope, a JEOL analytical electron microscope, a Cameca ion microscope, a Leybold-Heraeus laser microprobe mass analyzer, two laser Raman microprobes of NBS design, and extensive computer facilities, including a VAX $11 / 780$ computer and a DeAnza image processor.

Contact: Dale E. Newbury, B364 Chemistry Bldg., 301/975-3921.

Trace Gas Accurate measurement of gaseous

Measurement species is of paramount importance to a Techniques wide variety of industries in a number of applications ranging from the quantification of pollutant and toxic gas emissions to the quality control of products. The validity of data derived from such measurements is directly tied to the availability of useful gas measurement techniques and to the degree of understanding of their capabilities and limitations. While a variety of techniques have been applied to trace gas analysis, research directed at modifications, innovations, and rigorous assessments of these and new techniques is needed to improve the present state of the art in trace gas analysis. This research is particularly important to the growing requirements for the analysis of specific gas species in multicomponent gaseous mixtures and in the extension of accurate analyses to below the parts-per-million and parts-per-billion levels.

Current NBS research is focused on such trace gas measurement techniques as new detection systems using chemiluminescence, electrochemistry, infrared diode laser systems, capillary gas-liquid and gas-solid chromatography coupled to mass spectrometry, and isotope-dilution mass spectrometry Bureau scientists also are examining the use of class-specific detectors for gas chromatography and evaluating electronic circuitry to substantially optimize signal and reduce instrumentation noise and drift.

Contact: Walter L. Zielinski, Jr., B364 Chemistry Bldg., 301/975-3918.

A major task in the industrial production of organic fine chemicals, drugs and antibiotics, and biomolecules is the characterization of the product for both compositional analysis and quality control. Nuclear magnetic resonance (NMR) spectroscopy has proved to be a powerful and widely used tool for these and related purposes

One NBS program in this area involves the development of new and improved methods for the structural, quantitative, and conformational analysis of materials of chemical and biotechnological importance. The principal techniques that are being investigated include multınuclear. two-dimensional NMR and its combination with multiple quantum methods and distortionless enhancement by polarization transfer (DEPT). A specific goal of this work is the development of new twodimensional spectrum editing and filtering techniques and their application to struc. tural and conformatıonal characterization via correlations with multınuclear chemical shifts, coupling constants, relaxation
Nuclear Magnetic Resonance Spectroscopy of Bioorganics 


\section{Applied Mathematics}

Computational Designing comprehensive software for Mechanics modeling and database applications in materials science and engineering is the goal of this project. Current research on computer-aided modeling of the behavior of structural materials and components ranges from microscopic feature analysis of fatigue, fracture, phase change, creep, and polymer crystallization to the stresslife prediction of structural reliability of critical components based on mechanical and flaw-detection testing. In these studies, NBS researchers are emphasizing the interplay among the choices of data representation, appropriate physical principles, incisive mathematical and computational tools, and integrated software for graphics and databases. In addition, they are interested in mathematical theories of elasticity, plasticity, viscoelasticity, and so forth, as well as mathematical methods using matrix theory, finite-element algorithms, bifurcation analysis, statistical distribution concepts and sampling theory, and stochastic differential equations.

Contact: Jeffrey T. Fong, A302 Administration Bldg., 301/975-2720.

Measurement Quality assurance for physical and chemAssurance ical measurements and productivity improvement in laboratory operations depend on the development of new statistical methods and models. Current interests at NBS include experiment design for calibrations, robust estimation, time-series analysis, and graphical analysis. In addition, multivariate problems arise in the evaluation of complex electronic equipment. Therefore, estimation of variance components and of statistical tolerance limits for populations with several variance components are being studied.

Contact: Mary G. Natrella, A337 Administration Bldg., 301/975-2854.
Mathematical analysis, in combination with symbolic computation, leads to efficient analytical approximations by computers. Perturbation algorithms applied to nonlinear differential equations, especially in celestial mechanics, result in analytical developments whose complexity grows exponentially with the order of the approximation. Several different avenues are being explored to simplify literal developments generated by perturbation algorithms applied to nonlinear systems. These include the identification of algebraic structures on the domain of the normalization,

smoothing transformations to eliminate perturbation terms outside the kernel of the Lie derivative, preparatory transformations of a geometric nature, and the creation of natural intermediaries. Problems currently under examination at NBS are resonances at an equilibrium, perturbed pendulums, and the major theories of celestial mechanics. NBS researchers, using a LISP computer, are focusing their exploration on algorithms amenable to computer automation through symbolic processors.

Contact: Andre Deprit, A302 Administration Bldg., 301/975-2709.

Multi-dimensional geometric representations and data structures provide the basis for developing algorithms, software, and computer techniques used to describe, manipulate, and control objects handled in an automated process. NBS applications for this type of geometry include manipulating objects in automated design and manufacturing systems and optimal control of machining and mechanical processes. In addition, techniques of computational geometry are applied to computations with digitized cartographic data and to the display of quasi-crystal structures with icosahedral symmetries.

Contact: Christoph J. Witzgall, A302 Administration Bldg., 301/975-2725 or Sally E. Howe, A151 Technology Bldg., 301/975-3807.
Nonlinear Mechanics
Computational Geometry 
Vector To make effective use of vector

Algorithms and computers, new algorithms appropriate to

Mathematical the special architecture of these

Software machines need to be designed and incorporated in high-quality mathematical software for general use. Very successful vector algorithms have been developed for computations in numerical linear algebra (e.g., computation of the discrete Fourier transform), but few are available for combinatorial problems. One area of NBS research is concerned with developing vector algorithms appropriate to the combinatorial problems encountered in molecular dynamics simulations, in particular three-dimensional simulations with very large ensembles of particles. Additional areas include methods for solving highly nonlinear elliptic and parabolic partial differential equations as well as nonlinear constrained and unconstrained optimization problems and the solution of nonlinear systems of equations. NBS researchers also are working on the solution of integral equations and numerical evaluation of integrals. Software development goals include portable, interactive packages integrating modern graphics with numerical algorithms.

Contact: Francis E. Sullivan, A438 Administration Bldg., 301/975-2732.

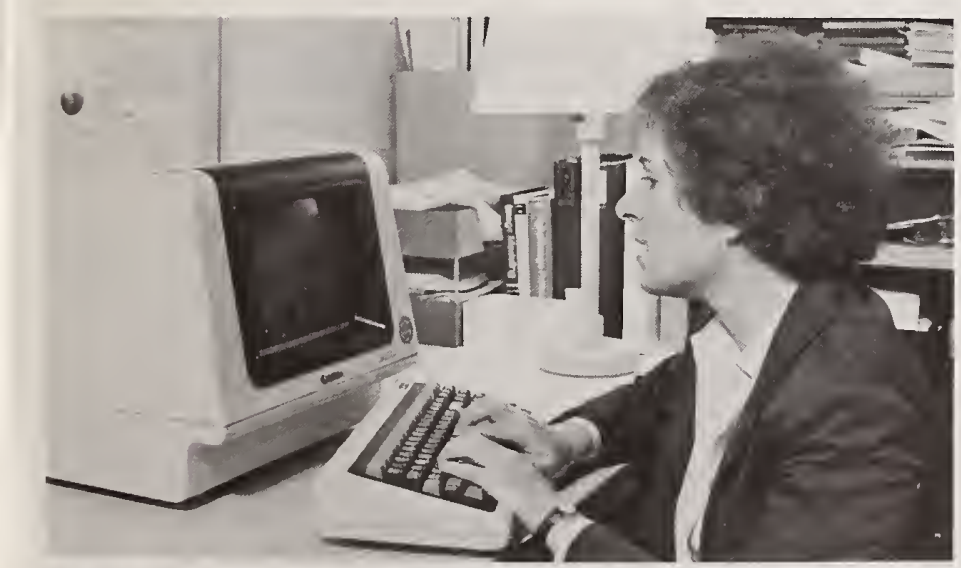

Mathematician Sally Howe is developing algorithms and software for robot motion planning that will be used in the Bureau's Automated Manufacturing Research Facility.
There is currently tremendous interest in the growth of gallium arsenide (GaAs) semiconductors on silicon substrates because such substrates can provide excellent mechanical strength and thermal properties that are not provided by the GaAs material alone. A major difficulty with growing $\mathrm{GaAs}$ on silicon has been the mismatch of lattice constants and the concomitant incorporation of defects. However, new studies show that a better match of the lattice $x x x$ can be achieved if the silicon substrate is cut at a small angle to the crystal plane. To probe the interaction of relevant semiconductor materials with the silicon substrate, NBS scientists are using sensitive, tunable laser sources to detect the individual states of the atoms and molecules as they interact with the growth substrate and growing material. Complete surface diagnostics and ultrahigh vacuum conditions are used in this work. NBS researchers are determining the sticking coefficients and adsorption energies of the individual spin-orbit states of gallium atoms and other dopant atoms plus the effects of vibrational excitation of the arsenic dimers on the growth of GaAs semiconductor materials.

Another Bureau study involves the deposition of thin films of amorphous silicon, which are used in photosensitive devices and in photovoltaic cells. Scientists are examining mechanisms involved in silane discharge and thermal chemical vapor deposition (CVD) production of such films. Mass spectrometry is used to study gas discharge processes, gas chemistry, and surface chemistry and their effect on film growth, character, and quality. Researchers are operating a threshold-ionization mass spectrometer that can detect $\mathrm{H}$ and $\mathrm{Si}_{x} \mathrm{H}_{n}$ radicals at densities of 1 part per million of the parent silane gases. The spectrometer also has a threshold sensitivity in the neighborhood of $10^{11} \mathrm{~cm}^{-3}$ in the reactor vessel. All electronics, vacuum, and gas handling apparatus necessary for examining if and dc discharges and thermal CVD under controlled conditions are available as is a pulsed laser for use in studying surface species by laser blowoff.

Contact: Stephen R. Leone, 303/4973505 or Alan C. Gallagher, 303/497. 3936, Div. 525, NBS, Boulder, CO 80303.
Studies of Semiconductor Materials 


\begin{abstract}
Advanced $\mathbf{A C}$ Thermal voltage and current converters Voltage and offer the most accurate and broadband Current method for measuring ac voltage and

Measurements current for applications in communications, power generation, aerospace, and defense. Thermal transfer standards are calibrated by NBS in terms of reference converters which have themselves been characterized by reference to the NBS primary standards. The primary standards are a set of special multijunction thermal converters whose performance is known over quite limited ranges of current, voltage, and frequency.

Complex, bootstrap methods are used to extend the current, voltage, and frequency ranges. However, because uncertainties expand considerably in those regions remote from the primary standards, NBS is studying new methods and converter designs to reduce significantly uncertainty for these regions. Of particular interest are voltages below 0.5 $V$ at all frequencies and frequencies from $100 \mathrm{kHz}$ to $1 \mathrm{MHz}$ at all voltages. The apparatus available for the studies includes the NBS primary standards with sub-part-per-million (ppm) uncertainty in a core region of parameter space and a number of high-accuracy comparators, both manual and automated, with subppm accuracy capability.
\end{abstract}

Contact: Joseph R. Kinard, 301/975-4250 or Norman B. Belecki, 301/975-4223, B146 Metrology Bldg.

Laser Many of the most sensitive and sophistiStabilization cated applications of lasers depend on the laser's spectral coherence, frequency stability, and low-intensity noise. For a number of years, NBS scientists have been working on appropriate laser control techniques in the domain of laser intensity stabilization, laser frequency stabilization, linewidth reduction with active control techniques, and several methods for producing quantitative laser frequency scans. In the latter category are two new systems. One, based on optical sideband production by broadband microwave phase modulation of the laser, allows scans over a $\pm 5-\mathrm{GHz}$ range with inaccuracy below $10 \mathrm{kHz}$. The other scan technique utilizes a novel interferometer/phase-locked rf system which maps optical frequency change into corresponding phase change of an rf signal suitable for control, stabilization, and scanning. This system is intrinsically free of errors which accumulate over large scans. In combination with a Lambdameter for coarse wavelength identification and a single absorption line used as absolute calibration, for example, NBS scientists can scan a few dozen angstroms with sub- $\mathrm{MHz}$ accuracy.

Another NBS system under development works entirely externally to a $\mathrm{cW}$ laser to shift the output laser frequency and reduce the intensity in a controlled manner. The first application of this is clearly a box which will sit in the output beamline of any laser and "eat" both frequency noise and amplitude noise in the intrinsic output of the laser. Bureau scientists have demonstrated this function by the combination of an external electrooptic phase shifter and an acousto-optic frequency shifter, which also functions as a fast controlled optical attenuator. Residual noise levels near the values fixed by the fundamental quantum fluctuations have been achieved in laboratory experiments.

Contact: John L. Hall, Div. 525, NBS, Boulder, CO 80303, 303/497-3126.

X-ray spectroscopy can provide information on electronic structure and on the local atomic structure of atoms in matter. Bureau scientists have constructed a synchrotron radiation beamline at the National Synchrotron Light Source which provides the highest flux, highest intensity, and highest energy-resolving power of any existing beamline in the x-ray energy range from $1 \mathrm{keV}$ to $5 \mathrm{keV}$. NBS equipment complements the synchrotron radiation instrumentation. X-ray absorption spectroscopy techniques such as $\mathrm{x}$-ray absorption near edge structure (XANES) and extended x-ray absorption fine structure have been used to determine the atomic structure of metals, semiconductors, polymers, catalysts, biological molecules, and other materials of interest to industry. The Bureau has additional experımental capabilities for
Study of Atomic Structure of Matter with $X$ Rays 
$x$-ray emission spectroscopy, $x$-ray photoelectron spectroscopy, and Auger electron spectroscopy, which are powerful probes of the electronic structure of solids, liquids, or gases.

The $x$-ray standing wave technique uses interference between incident and diffracted $x$ rays to determine the precise location of impurities or imperfections within a crystal or at its interfaces. The technique can be used to investigate the location of dopants and imperfections in semiconductors or optical crystals, growth of overlayers on crystals, and the structure of catalysts supported on crystal substrates. In addition, evanescent $x$ rays which penetrate only a few nanometers from an interface can be controlled to study chemical composition in the vicinity of an interface

NBS scientists recently pioneered a new technique, diffraction of evanescent $x$ rays (DEX), which combines and extends the capabilities of the $x$-ray standing wave method and experiments based on evanescent $x$ rays. The synchrotron radiation beamline provides an ideal facility for applying these techniques.

Contact: Paul L. Cowan, 301/975-4846 or Richard D. Deslattes, 301/975-4841, A141 Physics Bldg.

Vacuum and A wide variety of industries depend on Leak Standards accurate vacuum (pressure) and leak measurements for research and development and for process and quality control As part of its program in basic standards, NBS maintains, or is developing, pressure or vacuum standards from above atmospheric pressure to ultrahigh vacuum and leak or flow standards from $10^{-3}$ to below $10^{-9} \mathrm{std} \mathrm{cc} / \mathrm{s}$. Facilities for this research include five UHV systems, two low-range flowmeters, high-accuracy mercury manometers, pressure and vacuum control systems, and a variety of vacuum, electronic, data acquisition, and data analysis equipment. These facilities and measurement capabilities are used to develop improved measurement techniques and equipment and to investigate

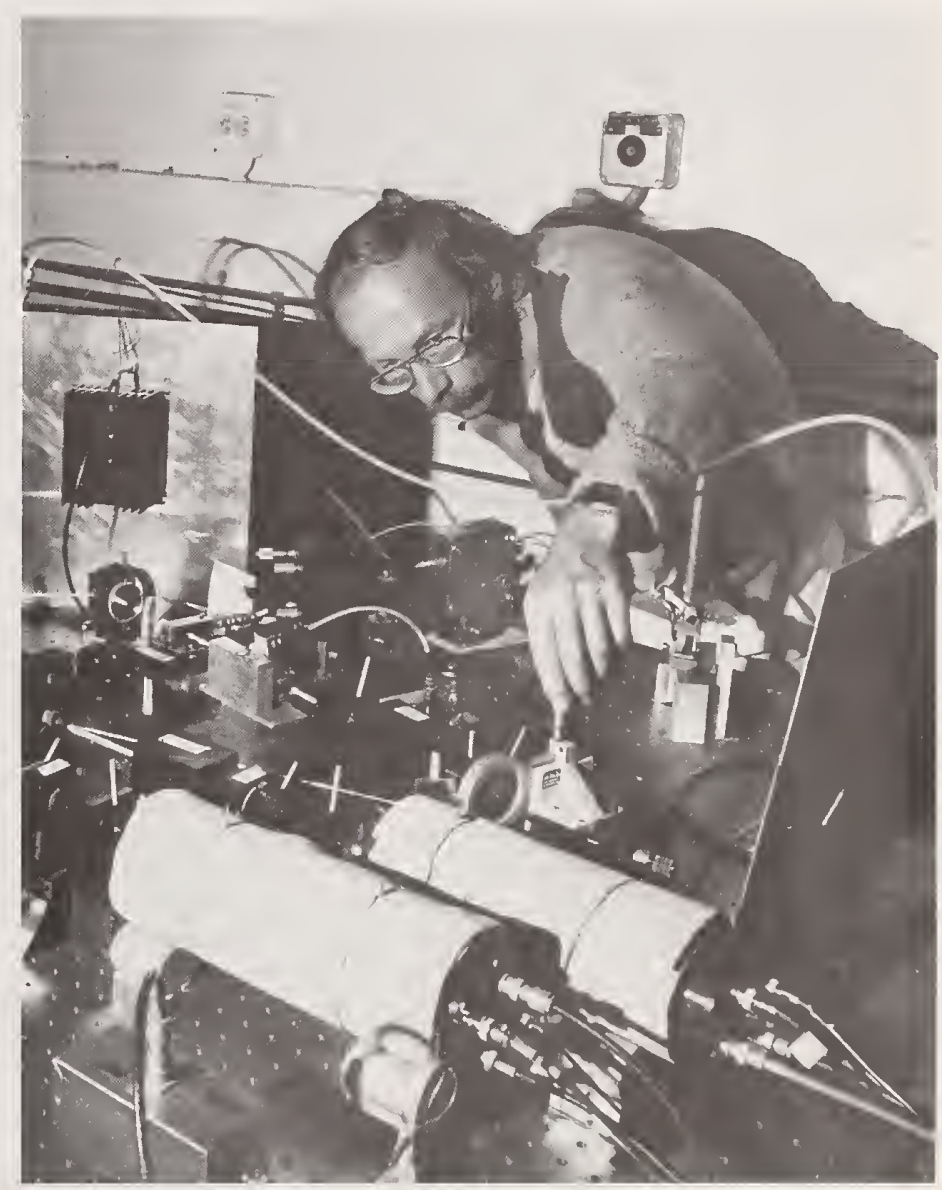

Physicist Dieter Hils, at the NBS/University of Colorado Joint Institute for Laboratory Astrophysics, conducts research in the stabilization of laser systems to provide standard sources of monochromic optical waves. Such devices are of commercial interest for many areas of industrial and scientific research.

the performance of vacuum and pressure equipment, including mechanical pressure gauges, momentum transfer gauges, ionization gauges, standard leaks, and residual gas analyzers. In addition, NBS plans to use this measure. ment capability to investigate material properties and physical phenomena of fundamental interest.

Contact: Charles R. Tilford, A515 Metrology Bldg., 301/975-4828. 
Frequency, Time, Advancements in communication and and Phase Noise navigation systems require atomic oscil-

Measurement lators with increased performance and reliability. NBS has several programs aimed at providing advanced frequency standards which have the potential for benefitting commercial atomic standards. Bureau scientists are currently working on an optically pumped cesium-beam standard which should significantly surpass the performance of standards based on magnetic state selection and detection. Their studies on ion storage and radiative cooling are probing the potential for standards operating at accuracy levels of one part in $10^{15}$ and perhaps beyond.

Aerospace systems often require extreme phase stability, which has led to a need for high-quality phase noise characterization amplifiers, frequency multipliers, oscillators, and other electronic components. Thus, NBS has initiated a program to develop methods for measuring phase noise in such components over a broad frequency range (into the millimeter range). The work will primarily involve the twooscillator technique, but other techniques will be studied.

Requirements for synchronization (time) and syntonization (frequency) of broadly dispersed sets of nodes for communication, navigation, and other electronic systems are increasing significantly. Because of the inherent reliability, simplicity, and low cost of using satellite transfer, NBS is studying several possible approaches for using this technique. The excellent performance of the NBS Time Scale and reliable ties to many other international timing centers provide the basis for analysis of the performance of these time-transfer techniques. In addition, the Bureau is equipped with GPS receivers, Earth communication terminals, and automated systems for statistical analysis of the performance of systems.

Contact: Donald B. Sullivan, Div. 724.03, NBS, Boulder, CO 80303, 303/497-3772.
Precision voltage standards based on solid-state references are finding increasing use in dc voltage metrology, particularly in the design and support of high-accuracy digital voltmeters and automated test equipment. To improve the quality of these standards, Bureau scientists are carrying out a research program on Zener diodes and similar devices. They are investigating device properties such as temperature coefficient, shortterm noise, long-term stability, and performance under temperature shock and power interruption. The aim of these evaluations is to identify devices suitable for a rugged 10- $\mathrm{V}$ transportable standard capable of providing sub-part-per-million $(\mathrm{ppm})$ accuracy when used as a transport standard between laboratories. The effects of temperature extremes, physical shock, and shipping delays on diode output is of special interest.

In another area of research, NBS is producing a stable 1.018- $\mathrm{V}$ output derived from a solid-state reference. The researchers are specifically interested in the design and manufacture of low-noise, stable resistors that are immune to temperature shock and power interruption. Facilities are available to test Zener diodes with $0.1-\mathrm{ppm}$ resolution and to manufacture both bulk metal foil and wire-wound resistors. Detailed study of the dependence of device performance on fabrication parameters is possible through high-accuracy measurements referenced to national standards and collaboration with Bureau scientists in several NBS divisions on the fabrication of Zener devices.

Contact: Bruce F. Field, B258 Metrology Bldg., 301/975-4230 or Norman B. Belecki, B146 Metrology Bldg. 301/975-4223.

High-precision mass measurements on mechanical balances in industrial standards laboratories are now very slow and labor intensive. Since fully automated mass measurement systems are not yet commercially available, the NBS mass program is developing a low-cost modular system. It will include a balance beam servo-system for automatic data acquisition; automatic modules to probe
Solid-State DC Voltage Standards 
air temperature, pressure, and relative humidity; a thermal "weight-soaking" plate servoed to the temperature of the balance chamber; a robotic weightchanger that can accommodate multipleweight combinations; and integrated software to control the measurement process and analyze the data with the NBS mass code. Many of these concepts have already been incorporated into a transportable mass measurement assurance package that is now being tested in industrial and state metrology laboratories. A fully automated system will not only reduce the labor cost of mass measurements, but will also minimize the operator and environmental biases that enter the manual measurement process.

Contact: Randall M. Schoonover, 301/9754216 or Robert D. Cutkosky, 301/9754214, B160 Physics Bldg.

Temperature Improved industrial processes and Sensor Research sophisticated scientific research require temperature sensors that cover wider temperature ranges with better accuracy and precision. For example, the degradation of thermocouples exposed to high temperatures for extended periods of time represents a serious impediment to temperature measurements in jet engines, furnaces, and so forth. NBS has several projects under way to test and improve the performance of currently available sensors. These sensors include thermocouples, platinum resistance thermometers, and a variety of cryogenic temperature transducers. The temperature range covered by these projects extends from cryogenic temperatures $(0.005 \mathrm{~K})$ to about $1500{ }^{\circ} \mathrm{C}$. For this research, the Bureau has excellent temperature calibration facilities, an automated laboratory equipped to evaluate thermocouples at high temperatures, several laboratories equipped for work on platinum resistance thermometers, and cryogenic laboratories where measurements can be made in the millikelvin range. NBS scientists are planning a series of studies of new materials, new principles, and new techniques to provide uniform and accurate temperature measurements.

Contact: Robert J. Soulen, B128 Physics Bldg., 301/975-4801.
In a strongly computer-oriented program, NBS researchers are seeking to develop an improved understanding of the relations among the chemistry, microstructure, durability, and other factors that affect the performance of cement, concrete, and other inorganic building materials. Current areas of research include mathematical modeling of the development of microstructure in cement hydration and of the effects of microstructure on cracking of concrete; investigations of mechanisms of materials degradation; and development of artificial intelligence systems for optimizing the selection of building materials. Bureau scientists are conducting analytical and experimental research on cracking and the dynamic properties of concrete and other brittle building materials. They are performing chemical and microstructural studies using a variety of techniques, including light microscopy, scanning electron microscopy, x-ray diffraction, and thermal analysis.

Cement hydration research contributes to making concrete a more predictable material. Therefore, NBS is seeking fundamental understanding of portland and related cements and their reactions with water by developing and validating mathematical models based on plausible reaction mechanisms, taking into account such processes as diffusion, nucleation, and growth of new phases. The models, which will probably be run on a Cyber 205 computer, will predict the courses and kinetics of the many reactions taking place during cement hydration and the development of microstructure under a wide range of conditions. Special experimental techniques employed in studying the reactions of cements include, in addition to the techniques mentioned above, microcalorimetry, pore solution analyses, ion chromatography, and Fourier transform infrared spectroscopy.

Contact: James R. Clifton, B348 Building Research Bldg., 301/975-6707.

Experimental and analytical research is under way at NBS to develop design criteria for steel and masonry structural components subjected to dynamic loads such as winds and earthquakes. The work involves identifying limit states and

\section{Cement and Concrete}




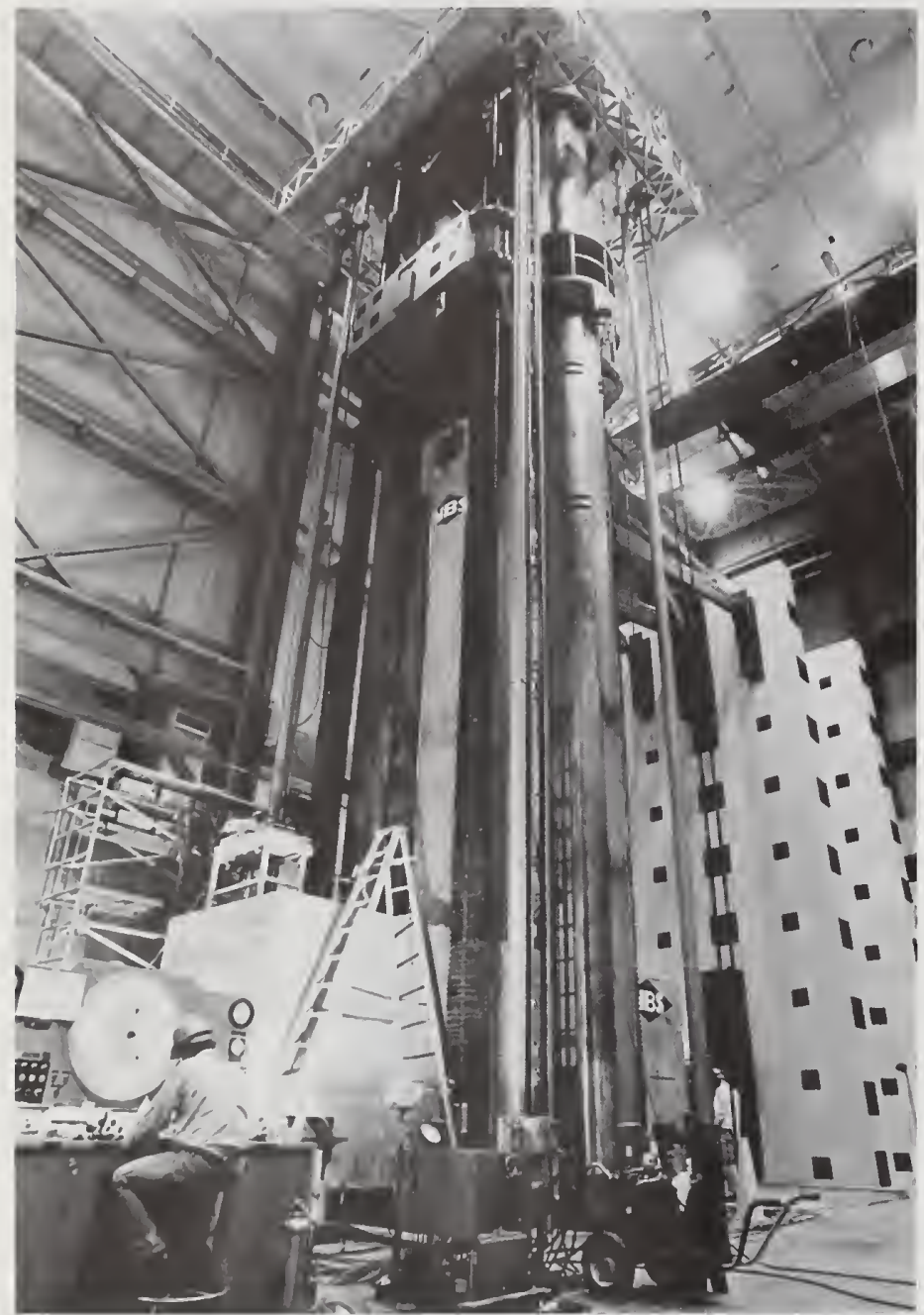

This computerized large-scale structural test facility is used to test the performance of full-scale bridge and building components during earthquakes.

establishing criteria to ensure structural safety. In the laboratory, NBS

researchers use a unique computercontrolled test facility that can impose forces and/or displacements in three directions simultaneously and a 12 million-pound capacity universal testing machine capable of testing large-scale structural components 60 feet tall. A high-performance computer graphics laboratory including a VAX computer system and specialized graphics-based finite element software is utilized for the analytical work.

Contact: Charles G. Culver, B268

Bullding Research Bldg., 301/975-6048.
To aid in the creation of buildings that operate more efficiently and better meet user requirements, NBS researchers are investigating the interaction between building occupants and illumination/daylighting systems. The work includes developing criteria for illumination quantity and quality and determining the effects of light source color and the color, size, and shape of the object on the performance of visual tasks. In addition, the research involves evaluating both the trade-offs and availability of daylighting as well as illumination controls and their interaction with building heating/cooling systems. The "basic" research laboratory consists of a multichannel electro-optical system that allows light to be delivered to the human eye in an experimentally reproducible manner, while the "applied" research laboratories provide a realistic environment for studying color rendering (distortion) of energy-efficient lighting systems.

Other research projects include daylight prediction computer modeling based on detailed simulation and experimental measurements. Also, the Bureau has initiated an experimental project to determine thermal interactions between lighting systems and HVAC (heating, ventilating, and air-conditioning) systems to provide improved design and evaluation techniques from the standpoint of energy effectiveness, luminous efficacy, and life expectancy of the lighting fixtures.

Contact: Belinda L. Collins, A313 Building Research Bldg., 301/975-6456.

NBS researchers are studying techniques for measuring and modeling indoor contaminants, specifically the effect of air leakage and ventilation systems on their levels and movements. NBS pioneered the measurement science for air infiltration by introducing the tracer gas technique. Currently the program has expanded into measurements and computer simulation modeling of various indoor air contaminants, notably formaldehyde and radon. Radon decay and dilution processes have been modeled, and several in-situ measurements are being undertaken to develop

\section{Illumination Engineering}


techniques to minimize excessive buildup of radon coming from the ground. Bureau researchers have investigated formaldehyde emission rates from pressed particle boards and other building materials to validate a prediction model developed by the Oak Ridge National Laboratory. In addition, NBS is developing comprehensive computer simulation models for predicting emission, dilution, absorption, and convective movement of contaminants in and through buildings with a number of rooms. An advanced gas chromatographic mass spectrometer is used to measure and analyze many indoor contaminants, especially the volatile organic compounds.

Contact: Richard A. Grot, B114 Building Research Bldg., 301/975-6431.

Organic Building As part of a program to increase the Materials usefulness, safety, and economy of building materials, NBS is conducting basic and applied research to determine the quality and predict the service life of organic building materials such as protective coatings for steel, adhesives, roofing materials, and plastics. Specific areas of basic research include advancing the knowledge of degradation mechanisms, developing improved characterization methods, and designing mathematical models of the degradation processes. The results of this research are then used to derive stochastic models for predicting the service life of these materials. The stochastic models have a basis in reliability theory and lifetesting analysis.

Bureau scientists are now using computer analysis of infrared thermographic images for studying the rates of corrosion and the formation and growth of blisters beneath opaque protective coatings. They are also utilizing gelpermeation chromatography, gas chromatography, and spectroscopy to study the mechanisms of photolytic and thermal degradation of poly (methyl methacrylate). In both projects, reliability theory and life-testing analysis techniques are used for predicting the service lives of the materials.

Contact: Larry W. Masters, B348 Building Research Bldg., 301/975-6707.
An extensive program to determine the performance of heat-pump and other noncryogenic refrigeration-cycle systems is under way at NBS in both the field and laboratory. The systems being studied include electric-driven, heatdriven (adsorption cycle), and heatengine-driven systems. An essential part of this program is the development of analytical models that will predict the performance of systems under transient as well as steady-state conditions and with single refrigerants and refrigerant mixtures. These models will be for specific systems, that is, engine-driven or electric-driven, with or without storage, in specific applications such as different buildings and climates. These models will predict the energy performance with various parametric changes, including component sizing

Contact: David A. Didion, B122 Building Research Bldg., 301/975-5881.

Bureau researchers are devising and improving methods for measuring the heat-transfer performance of thermal insulation and other materials used in the construction of buildings. An essential part of this program is the development of comprehensive analytical and mathematical models that will predict the performance of these materials under transient and steady-state conditions of use. These models will be developed for individual materials and for combinations of materials that are used in the design and construction of building walls, windows, doors, floors, ceilings, and roofs. The work is primarily of a laboratory nature, and excellent new facilities are in place for use in the program. They include a line-heat-source, guarded-hotplate apparatus that can accommodate specimens up to 15 inches thick with computerized control and data-logging and reduction capabilities, and a calibrated hot-box test facility to measure simultaneously air, moisture, and heat transfer characteristics through a full-size wall 10 feet high and 14 feet wide.

Contact: A. Hunter Fanney, B306 Building Research Bldg., 301/975-5864.
RefrigerationCycle-System Studies

\section{Thermal} Insulation 
Ceramic Sintering of ceramics is a complex

Processing process that involves the interaction of many different processing variables. The influence of these processing variables on sintering cannot be determined simply by measuring final density or some other end-point property. Chemical composition of the ceramic powders is known to be of major importance and, under certain conditions, can mask effects of most other processing variables. As part of a program on ceramics, NBS scientists are investigating the effect of trace levels of impurities using clean-room processing to produce, compact, and sinter ultrahigh purity ceramics. Compositions will be measured at different stages of the process using nuclear activation analysis (gamma-ray spectroscopy). Results from this program will enhance investigations of other processing variables, including particle size, shape, agglomeration, compaction method, and atmosphere under controlled composition conditions. Such data will lead to better models for microstructure evolution during sintering. Use of predictive models, in conjunction with other ongoing efforts to produce unique compositions and phases, can lead to new advanced ceramic materials, with unique microstructures and properties.

Contact: Edwin R. Fuller, Jr., A258 Materials Bldg., 301/975-5795.

Tribology of NBS is examining the wear and frictional Ceramics characteristics of advanced ceramic materials with state-of-the-art wear test analysis methods. Studying hightemperature ceramics, especially the type necessary for ceramic heat engines, requires new test procedures and techniques and standardized methodologies. For such research, friction and wear tests may be performed over a wide temperature range with a controlled test environment. Equipment is presently available at the Bureau to test ceramics from 20 to $1500{ }^{\circ} \mathrm{C}$, and a unique high-temperature, controlled-atmosphere ceramic wear test facility that will produce temperatures up to $1500{ }^{\circ} \mathrm{C}$ is almost ready for use.

Bureau researchers are also investigating the control of friction and wear by advanced lubricants and lubricant transfer methods. Pre- and post-test analyses include wear debris analysis, profilometry, and scanning electron microscopy (SEM). SEM facilities are used to examine wear tracks and debris.

Contact: Ronald G. Munro, A215 Metrology Bldg., 301/975-3671.

Imperfections in highly perfect crystals typically limit their performance in hightechnology applications such as optical communications and optical signal processing. In particular, limitations in the perfection of electro-optic and photorefractive materials such as lithium niobate and bismuth silicon oxide have severely inhibited the development of optical switches and modulators. Bureau researchers are investigating crystal perfection at a unique, monochromatic $x$-ray topography facility at the NBS/Naval Research Laboratory beamlines on the high-energy ring at Brookhaven National Laboratory's National Synchrotron Light Source. The information from current studies, conducted jointly with growers of high-quality crystal, is expected to improve substantially the quality, and hence the performance, of these crystals.

Contact: Bruce W. Steiner, A329 Materials Bldg., 301/975-6122.

Bureau scientists are conducting several long-range programs on the fracture behavior of two types of ceramic materials, ceramic composites and polycrystalline ceramics. The program in composites focuses on determining the effects of the fiber/matrix interface on fracture behavior of ceramic matrix composites. The fracture studies on polycrystalline ceramics are exploring the effects of grain boundary compositions and structure on strength and stress corrosion susceptibility as well as the effects of phase transformations on the strength and fracture toughness of piezoelectric and dielectric ceramics. Facilities for these programs include mechanical testing machines for tensile and compressive tests, a microindentation hardness tester, and an environmentally controlled apparatus utilizing double beam configuration for environmentally enhanced crack growth studies.

Contact: Stephen W. Freiman, A329 Materials Bldg., 301/975-5761.

\section{Electro-optic Crystals}

Fracture of Ceramics 
Materials Utilizing microorganisms to extract Bioprocessing extraneous materials is one goal the steel, mining, minerals recovery, and waste treatment industries have in common. Biological leaching is a lowcost alternative to expensive energy processes. Therefore, NBS researchers are investigating microbiological processes for iron ore and scrap steel beneficiation through biosolubilization of copper and phosphate/silicate minerals, and bioprocessing - leaching and recovery - of strategic metals by mesophilic and thermophilic microorganisms. To develop appropriate processes for each type of extraction, characteristics of such microorganisms must be understood. Thus, NBS is developing and analyzing nondestructive, surface molecular analysis methods including microscopic absorption and emission spectroscopies. In addition, Bureau scientists are using ultratrace molecular speciation methods for solution and gas phase chemical speciation of diagnostic products or intermediates (gas and liquid chromatographs coupled with graphite furnace, flame photometric, and mass spectrometric detectors). Other areas under study include engineering designs, bioreactor systems, and development of standards such as metal sulfide Standard Reference Materials and ore bioleaching test protocols.

Contact: Gregory J. Olson, 301/9755637 or Frederick E. Brinckman, 301/975-5737, A329 Materials Bldg.

Ceramic Powder Improved characterization of particle size, Characterization shape, and crystal chemistry of ceramic powders is now recognized as important to the development and production of high-quality ceramics for advanced technological applications. To obtain precise and reproducible measurements of particle characteristics, Bureau scientists are utilizing a variety of instrumental methods, including scanning electron microscopy with image analysis. Theoretical investigations and statistical analyses of data are then used to compare the results of the measurement methods.

The broad NBS program also includes development of $\mathrm{x}$-ray diffraction techniques, reference materials and databases for characterizing ceramic starting powders, and ceramic monoliths. Automated $x$-ray diffraction instrumentation with position sensitive detectors has the potential for rapid, production-line characterization of both phase composition and material properties of powders and monoliths. Characterization includes determination of powder composition (qualitative and quantitative phase and chemical constituents), glass-to-crystalline ratio, crystallite and particle size, residual stress, lattice parameters, and thermal expansion. Special equipment available for studying the relationships between particle characteristics and processing parameters includes SEM, STEM, DTATGA, furnaces with atmospheric control, three automated powder diffractometers with high-temperature stage, coulter counter, BET, sedigraph, and particlesize classifiers.

Contact: Alan L. Dragoo, 301/975-5785; Carl R. Robbins, 301/975-5786; or Camden R. Hubbard, 301/975-6121, A258 Materials Bldg.

Techniques that probe the surface environment of powders during crystallization and compaction are likely to yield significant information on the link between observed reaction chemistry and product quality. To this end, NBS is investigating Fourier transform infrared (FTIR) and electronic absorption/emission spectroscopies for ceramic surface analysis. Two lines of research in progress exemplify the Bureau's approach and serve as models for further exploration. One project involves silicon nitride powders from various sources that have been examined by FTIR. Compositional purity (Si-N/Si-O ratio) and percent beta phase are easily assessed by this nondestructive technique. The other project concerns a fluorescent tag that has been developed for the siloxy group. The complex formed between the tag and $\mathrm{SiO}^{-}$on silica gel has been detected by epifluorescence microscopy imaging. This technique is expected to be useful in quantitating the chemistry occurring at crack tips in silica samples.

Contact: Robert A. Faltynek, 301/9755636 or Frederick E. Brinckman, 301/975-5737, A329 Materials Bldg.

\section{Ceramic Surface Analysis}


Optical Films Bureau scientists are analyzing the relationship between processing, structure, and properties of optical films grown by electron-beam (e-beam) deposition, e-beam coevaporation, and ion-assisted e-beam deposition. To support industrial development of new optical materials with superior properties such as decreased porosity, decreased stress, greater index stability, and lower absorptivity, these researchers are studying the evolution of film structure starting from nucleation, growth, thin-film adhesion, and thermal properties of films. Characterization capabilities include refractive index by $\mathrm{m}$-line spectroscopy and channel spectra, thickness, absorption coefficients by laser calorimetry, stress by interferometry, thermal diffusivities by photothermal radiometry, $x$-ray photoelectron spectroscopy, Raman spectroscopy, extended $x$-ray absorption fine structure, $x$-ray diffraction, and electron microscopy. New areas to be pursued, with impact on optical signal processing technologies, are epitaxial growth and modulated structures for light-guiding optical films with enhanced electro-optic and nonlinear optical properties.

Contact: Albert Feldman, A329 Materials Bldg., 301/975-5740.

Ceramic Phase Ceramic phase equilibria studies at NBS Equilibria involve complementary research activities in experimental, theoretical, dataevaluation, and compilation aspects of ceramic phase equilibria. These studies include all classes of inorganic materials except alloys. The data-evaluation and compilation work is carried out under the joint American Ceramic Society

(ACerS)/NBS program to provide industry and others with a comprehensive database of up-to-date, critically evaluated phase diagram information.

The established, broad-based, fundamental and applied high-temperature chemistry research program examines chemical phenomena temperatures from 25 to above $5000{ }^{\circ} \mathrm{C}$, atmosphere pressures from $10^{-10}$ to $20 \mathrm{~atm}$, and multicomponent solid, liquid, and vapor systems. New or improved measurement techniques are needed for identification and quantification of species and components present under these conditions.
Furthermore, transitional species, such as clusters of atoms or molecules, present a particularly unique measurement challenge. Researchers need to measure such species to be able to understand condensation and surface phenomena on a molecular-level and to develop materials with unique structures and properties. In addition to experimental work, the research involves computer modeling to extend the laboratory-based data to industrial-process conditions.

Contact: John W. Hastie, A329 Materials Bldg., 301/975-5754.

Bureau scientists are investigating lowtemperature synthetic approaches to both oxide and non-oxide ceramic powders and the use of neutron and $x$-ray scattering expertise in atomic and microstructural studies. Special emphasis is placed on the use of novel chemistry and techniques to solve the problems of generating homogeneous, multicomponent materials with predictable properties. In general, systems currently under study include barium polytitanates, zirconiatoughened aluminas, and silicon carbidetitanium carbide particulate composites

These researchers are examining the molecular structure of species in multicomponent alkoxide systems, the kinetic aspects of sol-gel reactions, and to the microstructure of product powders and glasses. These studies were undertaken to define key process variables and guidelines for developing practical sol-gel processes. Coupled with powder synthesis and subsequent compaction is the development of in-situ measurement techniques, including small-angle neutron and small-angle $x$-ray scattering. Such techniques will allow characterization of key fundamental parameters controlling early stages of the material processing. This effort complements nondestructive evaluation (NDE) sensor development so that industry will be able to detect flaws at the very early stages of processing where the raw material can be recovered and treated.

Contact: Kay A. Hardman-Rhyne, A329 Materials Bldg., 301/975-6103.
Ceramic Chemistry 
High-Temperature The success of structural ceramics in

Reliability of high-temperature applications, such as

Structural heat engines or heat exchangers,

Ceramics depends on the reliability of these materials at temperatures that approach their thermal limit. Because creepinduced cavitation is the primary cause of failure at these temperatures, the characterization of nucleation, growth, and coalescence processes associated with cavitation is essential to establish long-term reliability. Bureau scientists are studying basic processes associated with creep cavitation on a variety of ceramic materials. To develop techniques for predicting component lifetime, they are relating damage accumulation during creep to mechanisms of creep and creep-rupture. This research includes creep and creep-rupture measurements at temperatures as high as $1600^{\circ} \mathrm{C}$, and the use of transmission electron microscopy to evaluate modifications in the microstructure that result in the generation of strength-limiting defects.

Contact: Sheldon M. Wiederhorn, A329 Materials Bldg., 301/975-5772.

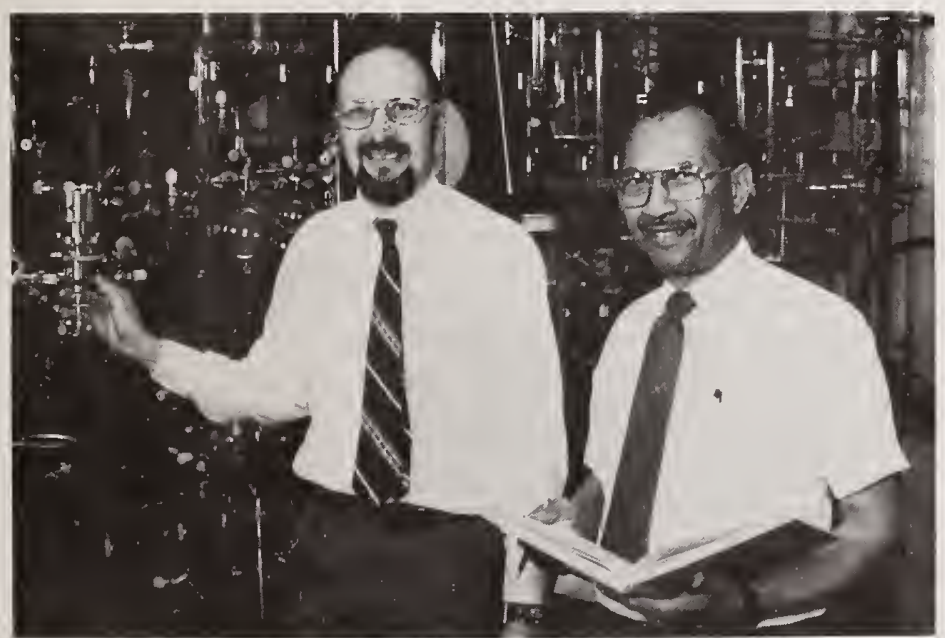

Research chemist Joseph Ritter (left) and physical science technician Norman Adams are shown with the reactor they designed for synthesizing advanced ceramic powders at low temperatures.
Improved energy and manufacturing effi- Separations ciencies could result from using various techniques to separate and recover desired products from fluid chemical mixtures. To improve separation procedures, the Bureau is creating a science base for a variety of separations. As part of this project, scientists are working in three rather diverse areas. One project involves the aqueous two-phase partitioning of proteins and biomolecules, including measuring mass transfer characteristics and diffusion coefficients and developing mass transfer correlations for classes of biomolecules. In another project, NBS researchers are studying membrane separations of carboxylic and amino acids from reaction products and waste streams using ion exchange membranes. This research includes separating organic compounds from polar solvents and developing models for the transport of weak and strong electrolytes through cation exchange columns. Work in these two areas is expected to impact the commercialization of products in the burgeoning biotechnology industry.

In a third study, Bureau scientists are separating gas using novel/efficient separation methods such as facilitated transport through liquid membranes, while conducting complementary modeling activities. They are currently working on acid-gas separations using ion exchange membranes.

Contact: Subhas K. Sikdar, 303/497-5232; J. Douglas Way, 303/497-5298, or Richard D. Noble, 303/497-3416, Div. 773.10, NBS Boulder, CO 80303.

Thermophysical properties data are essential for the design and operation of many chemical processes such as supercritical extraction. To obtain these data, NBS scientists are using three new phase equilibria apparatus for studies at elevated temperatures. One has been used to perform VLE measurements on carbon dioxide-hydrocarbon systems and refrigerant-hydrocarbon systems, the second is a dew/bubble point apparatus extending to $800 \mathrm{~K}$, and the third employs a palladium-silver membrane to measure the fugacity of hydrogencontaining mixtures. Four exceptional instruments are available for making PVT, -PVTx, and heat capacity measurements
Properties of Fluids 
on pure fluids and fluid mixtures: an isochoric PVT apparatus, a combined Burnett/isochoric PVT apparatus, a magnetic suspension densimeter for PVT and PVTx measurements, and an instrument to measure constant volume heat capacity. In addition, an apparatus for sound speed measurements is available.

Bureau researchers are also devising techniques to characterize fluids and fluid mixtures when the temperatures, pressures, and times involved can result in reactions during the measurement process. Included in this work is a wellequipped analytical laboratory and specialized apparatus to study reactions.

Contact: Thomas J. Bruno, 303/497-5158; James E. Mayrath, 303/497-3553; William M. Haynes, 303/497-3247; or Gerald C. Straty, 303/497-3502, Div. 774.03, NBS, Boulder, CO 80303.

Radio Frequency The successful processing of paper pulp, Wave Diagnostics flour, coal slurries, and sludge depends of Disperse on accurate measurements of the Systems percent of solids suspended in water in a pipeline. To help improve these measurements, NBS is studying the propagation of radio waves inside a conducting pipe filled with complex materials. One result of this research has been the successful measurement of the solids fraction in a two-phase water-solid slurry. This was accomplished by measuring the wavelength and attenuation of radio waves in a pipeline utilizing a 32-element antenna array placed along the axial direction. From the known frequency and wavelength the speed of the radio wave, and hence the dielectric constant, can be deduced. By then applying a mixing rule and the dielectric properties of the individual components researchers can determine the solids fraction in a slurry. NBS is extending the project to incorporate systems with axially varying dielectric constants such as adsorption columns and settling suspensions. Other measure ments such as polarization rotation in a swirling slurry flow and Doppler shifts due to fluid flow are being planned.

Contact: Adolfas K. Gaigalas, 109 Fluid Mechanics Bldg., 301/975-5941.
The accelerating costs of scarce fluid resources - particularly gas - are producing increased concerns about the performance levels of gas meters. For these reasons, improved flow measurement traceability needs to be established and maintained so that realistic, quantified data are generated on a continuing basis to assure practical gas measurements at satisfactory specified levels of performance. To achieve the desired flow measurement traceability, the Bureau is designing transfer standards to link the performance of calibration facilities to appropriate national reference standards. As part of this program, NBS researchers plan to develop a fluid measurement transfer standard and evaluate its performance.

Because of the importance of these measurements, transfer standards need to be designed so that high levels of confidence can be placed in them and their performance. The new transfer standards will be rigorously evaluated against NBS gas flow calibration standards. This evaluation phase of the proposed program will involve the appropriate range of calibrations to be done on the developed standards so that performance levels can be assured at specified levels.

Contact: George E. Mattingly, 103 Fluid Mechanics Bldg., 301/975-5939

NBS researchers are gaining a fundamental understanding of chemical processes in fluidized bed reactors in the areas of bioprocessing and advanced optical measurements at high temperatures. Their work in bioprocessing is aimed at improving the design and operation of liquid fluidized-bed and expanded-bed bioreactors for industrial fermentation processes. To demonstrate the feasibility of monitoring biofilm development in fluidized-bed fermenters, they plan to investigate the effects of hydrodynamics on the development and detachment of immobilized-cell layers from individual carriers, and the effects of these layers on carrier-particle hydrodynamics, using magnetically suspended spheres and other techniques.

\section{Flow} Measurement Transfer Standards
Fluidized Bed Reactors 
Research in the high-temperature area is based on a fluidized-bed reactor that is being adapted for advanced in-situ optical measurements at temperatures up to $1150^{\circ} \mathrm{C}$. The principal advance beyond the state-of-the-art probing of fluidized beds has been the design of an optical probe that allows observations at one or more arbitrarily chosen locations in the interior of the bed. Presently, NBS is interested in studying solids movement in fluidized beds based on the fact that a burning particle generates a distinct signal as it arrives at an optical probe location. The goal of this work is to advance the understanding of the mixing problem in multiphase chemical processes.

Contact: Andrej Macek, B312 Physics Bldg., 301/975-2610.

Thermophysical Chemical process technology requires an and Supercritical accurate knowledge of various ther-

Properties of mophysical properties of pure, polyfuncMixtures tional chemicals and their mixtures. NBS scientists conduct research on the most important of these properties - equilibrium phase composition, density, and enthalpy. One NBS project deals with developing predictive methods for the properties of chemically dissimilar compounds. Of special interest are those compounds found in gas and coal processing as well as chemical systems. Bureau researchers are planning related experimental phase equilibria studies.

A second project is a combined experimental and theoretical study aimed at developing accurate predictive models for the thermodynamic and transport properties of supercritical solvent mixtures. The work includes PVTx and VLE measurements on mixtures containing carbon dioxide, halogenated hydrocarbons, and similar supercritical solvents. Other experimental work includes the use of supercritical chromatography to measure diffusion coefficients in supercritical mixtures while theoretical studies focus on the application of extended corresponding states to supercritical systems.

Contact: James F. Ely, 303/497-5467; Joseph W. Magee, 303/497-3298; or Thomas J. Bruno, 303/497-5158, Div. 774.03, NBS, Boulder, CO 80303.

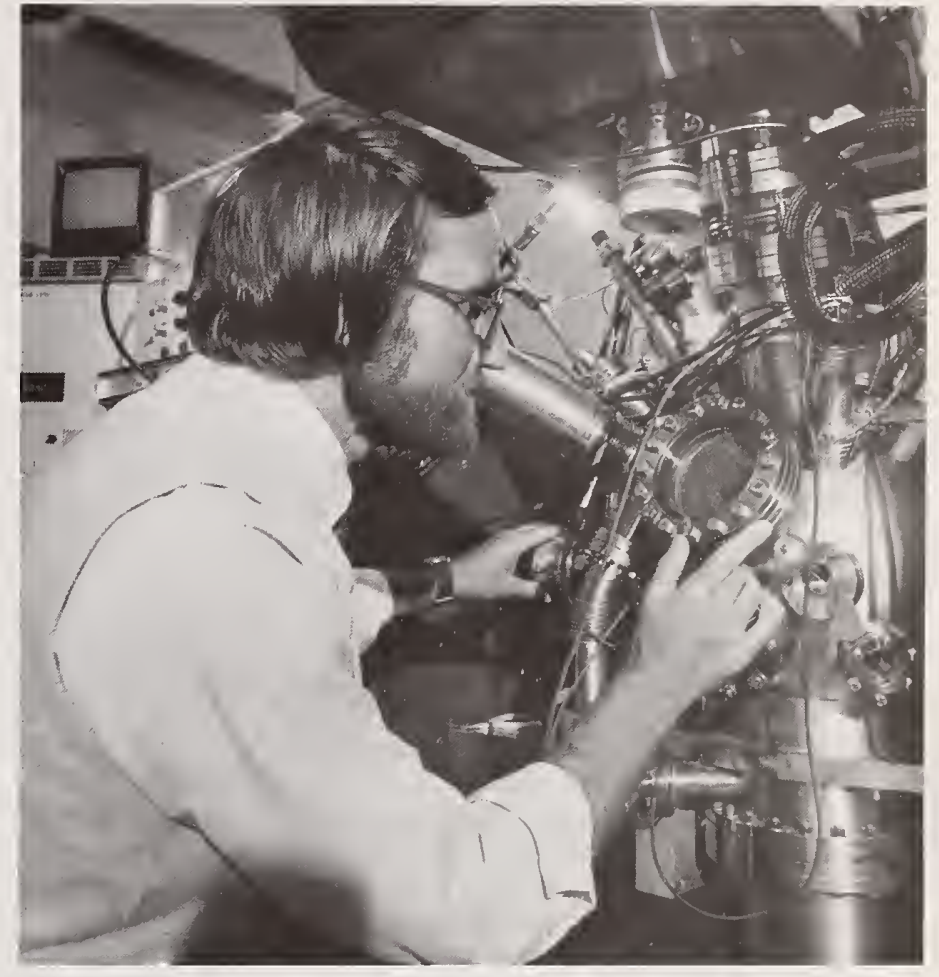

Through the use of very thin films and detailed surface analysis, physicist Stephen Semancik and his colleagues have characterized the surface adsorption properties of tin oxide. Such information can be used to improve tin oxide sensors.

To understand performance of thin-film and chemical sensors, NBS is performing

Chemical Sensor experimental and analytical research related to these types of sensors. Such studies are expected to improve the accuracy, stability, selectivity, and response of chemical sensors and to stimulate ideas for entirely new measure ment techniques. The Bureau's activities incorporate adhesion, oxidation, interlaminar diffusion, surface adsorption and desorption, and phase morphology to uncover mechanisms of chemical sensing. Analytical methods relate the structure and composition of sensing devices to fabricating parameters and performance. Sensors include thin-film thermocouples, fiber optic thermometers, thin-film resistance devices, moisture sensors, $\mathrm{pH}$ sensors, and gas detectors. Fabrication facilities include rf and dc sputtering for both alloy and reactive film deposition and gas reactors. 
To determine the feasibility of thin-film systems as chemical sensors, NBS scientists are investigating the electronic properties of sputter coatings including dielectric strength, surface conductance, and capacitance. To examine surfacesensitive techniques, they are employing $x$-ray and uv photoemission, thermal desorption spectroscopy, SIMS, and insitu electrical measurements to characterize appropriate chemisorptive systems.

Contact: Kenneth G. Kreider, 301/9752619 or Stephen Semancik, 301/9752606, B312 Physics Bldg.

\section{Optical Sensing Recent advances in molecular biology}

in Bioreactors have led industry to recognize the potential benefit of applying these advances in a diversity of industrial sectors, such as the production of new drugs, food additives, and chemicals and conversion of biomass. However, implementation of these new technologies requires measurement capabilities which do not currently exist. Bioprocesses require a very closely controlled environment, normally realizable only in batch processes. This is mainly due to the complex kinetics of most bioreactions, separate periods of growth and product formation, biocatalyst degeneration, contaminant risks, and mechanical difficulties of handling rheologically complex material. Therefore, development of new on-line measurement techniques is critical for future implementation of bioprocesses. Furthermore, nonintrusive measurement methods are desirable to avoid difficulties associated with sampling, contamination, and long response time.

To help solve some of these problems, NBS scientists are developing optical techniques, especially with the use of tunable lasers, for making nonintrusive, rapid, and selective measurements. The use of fiber optics also permits the development of probes which are sterilizable, can provide measurements with good spatial resolution, and can be multiplexed to provide multipoint monitoring capability. Laser-induced fluorescence is being used to determine characteristics of amino acids, dipeptides, polypeptides, and esters while Raman and resonance Raman scattering will be utilized to provide more species and selectivity. Fluorescence techniques are also expected to provide a powerful tool for cell activity and intracellular kinetics measurements.

Contact: Hratch G. Semerjian, B312 Physics Bldg., 301/975-2609.

To minimize the cost of high-price fuel, U.S. industry wants to obtain maximum energy output from fuel combustion. Bureau researchers are tackling this problem by attempting to improve combustion efficiency. To investigate droplet vaporization, pyrolysis, combustion, and particulate formation processes and to delineate the effect of chemical and physical properties of fuels on the above processes, they are studying the dynamics of spray flames. Results of this study will provide an experimental database, with well-defined boundary conditions, for developing and validating spray combustion models. The experiments are being carried out in a spray combustion facility, with a moveable-vane swirl burner, which simulates operating conditions found in practical combustion systems. A combination of nonintrusive probing techniques is being used to obtain comprehensive data on the spray combustion characteristics, including soot particle and droplet size, number density and volume fraction, gas composition, and velocity and temperature fields.

Currently Bureau scientists are focusing their efforts on laser scattering and laser Doppler velocimetry measurements, which are being used to determine the correlation between droplet size and velocity distributions, respectively, in both low-temperature and burning sprays.

Contact: Hratch G. Semerjian, B312 Physics Bldg., 301/975-2609.

\section{Particulate and Droplet Diagnostics in Spray Flames}


Properties of A wide array of experimental and theoretInterfaces ical tools are being applied to obtain practical and fundamental understanding of the structure, properties, and effects of interfaces between fluid phases. NBS scientists are studying a number of problems in this area ranging from the conceptually simple to measure and/or calculate the interfacial tension between a liquid and its vapor, to more complicated ones such as the phase behavior of surfactant mixtures at water-air interfaces, the thicknesses of adsorbed liquid layers at solid-gas interfaces, the stability of foams, and the evolution of mists. Bureau facilities include an automated ellipsometer and an automated film balance, quasielastic light scattering for studying the evolution of mists, an apparatus for measuring foam stability, and simple setups for measuring interfacial tension. Computing facilities include a CDC 855 , a Cyber 205 vector processor, software, and experience in simulation of fluids.

Bureau scientists are planning research in several areas. One project will involve applying quasielastic light scattering to the measurement of interfacial tension of liquid-vapor mixtures at high pressure and the measurement of the dynamics of wetting layers. Another project will focus on measuring the permeability, surface viscosity, and surface diffusion coefficients of surfactant layers. In addition, the researchers will test NBS theories developed for the large increase of the surface tension of a liquid-vapor interface when it forms in a confined geometry (such as in a pore within a pellet) and for the large effects of ions on the thicknesses of adsorbed layers. They will also study the evolution of the drop size distribution of simple, but non-trivial, mists comprised of two hydrocarbons suspended in their own vapor.

Contact: Neil Olien, 303/497-3257, Div. 774, NBS, Boulder, CO 80303
The study of chemical kinetics at NBS has numerous applications, including controlling and monitoring environmental pollution, of interest to industry, energy, defense, and standards experts. Bureau scientists have been determining the kinetics and thermochemistry of industrially important organic molecules. molecular ions, and free radicals. Polycyclic aromatic compounds are important as reactants in coal-conversion processes and as products in virtually all pyrolytic reactions of organic substances. Facilities are available at NBS to determine the elementary rate constants and mechanisms for reactions which control the formation and destruction of aromatic compounds at elevated temperatures. These facilities can be used to study reactions involving polyaromatic free radicals in a variety of physical environments including high-temperature liquids, very low-pressure gases, and graphite surfaces. Complementary theoretical investigations also are possible. Other research involves the thermochemistry of ion-neutral interactions using variable temperature, high pressure, and ion cyclotron resonance spectrometry. In addition, a new laser laboratory incorporating the technique of multiphoton ionization mass spectrometry is available for state-of-the-art detection of free radicals-particularly biradicals. Other chemical dynamics studies can be carried out using picosecond spectroscopy.

Contact: Stephen E. Stein, A147 Chemistry Bldg., 301/975-2506.

To evaluate the properties of important biological building blocks such as proteins and nucleic acids, NBS recently began biothermodynamics studies to evaluate existing thermodynamic data. Biomolecule crystals, which can be purified and grown at the Bureau, can be examined by $x$-ray and neutron diffraction techniques for single crystals.

NBS researchers have developed quantum chemical computation techniques to calculate how complex systems of organic moiecules interact with metal ions in metalloenzymes. Computing facilities include an IBM 4381, an Evans and Sutherland color graphics system for model building, and the NBS Cyber

\section{Chemical \\ Kinetics and \\ Thermochemistry}

Biotechnology 


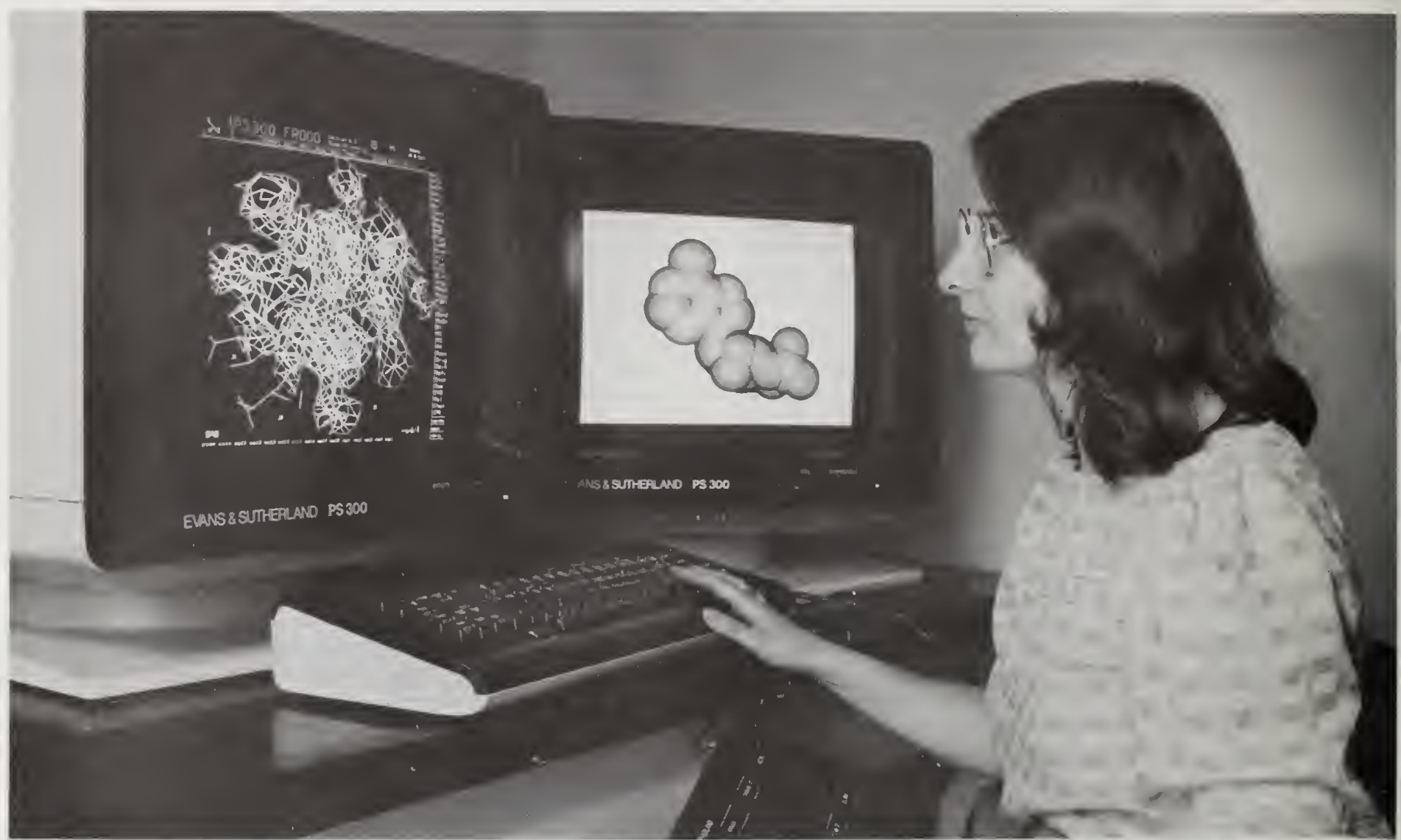

Using experimental data and sophisticated computers, physicist Irene Weber maps the detailed chemical structure of biomolecules to determine how they can be tailor-made to carry out specific reactions.

$855 / 205$ supercomputer. In addition, the thermochemistry of enzyme-catalyzed systems can be studied using a variety of calorimeters including micro-calorimeters designed and constructed at NBS.

Contact: Stanley Abramowitz, B348 Chemistry Bldg., 301/975-2586.

Surface Surface characterization is now an Characterization integral part of many technologies and industries such as catalysis, coatings, corrosion, semiconductors, computers, automobiles, and communications. The Bureau has several surface characterizatıon facilities available, including the use of synchrotron radiation at the NBS synchrotron ultraviolet radiation facility (SURF II) and at the Brookhaven National Synchrotron Light Source. A full complement of surface spectroscopies are avail- able at SURF II and in other NBS

laboratories. These can be used to identify molecular species and structures on surfaces. In addition, reaction chambers are connected to some for investigation of surface composition and catalytic reactions. New atom probe field ion microscopes offer high-resolution surface and depth-profile analysis capabilities. Moreover, spectrometers are available at the NBS reactor to perform neutron inelastic scattering and time-of-flight experiments. Neutron scattering is used to study higharea catalytic materials under highpressure reaction conditions. The sensitivity of this technique to hydrogen makes it particularly attractive for industrial chemical process studies.

Contact: Cedric Powell, B248 Chemistry Bldg., 301/975-2533. 


\section{Computer Sciences and Technology}

Personal The personal computer has become an

Computer omnipresent element in today's business

Security and scientific environment. However, as more sensitive and critical information is processed and stored on such systems there is an increasing need for methods to protect that information against unauthorized access or modification. The selection of information security measures, however, should be based on an analysis of the cost of such measures and the resulting reduction in losses NBS researchers are investigating various technologies that can be employed to achieve additional control and security of information on personal computer systems. Their research involves the identification, analysis, development, and application of these technologies.

While it is desirable to have security mechanisms as an integral part of computer systems, this is not always possible or economical because such mechanisms are often not part of the original system design. Therefore, NBS researchers also are examining the technology available to enhance the security of existing systems. This research involves identifying, analyzing, and comparing security mechanisms used in isolation or combination.

Contact: Allen L. Hankinson, B266 Technology Bldg., 301/975-3289.

Database Testing To improve the management of information resources, NBS researchers are developing test methods and techniques to be used in evaluating implementations of network database language (NDL), structured query language (SQL), information resource dictionary system (IRDS), and data descriptive file (DDF) for conformance to the emerging federal, national, and international standards. Researchers will attempt to derive a general methodology for designing conformance tests, to use this methodology to generate test suites, and to evaluate the test suites for effectiveness. A prototype implementation of the IRDS specifications will be used in this project. It may be suitable for such tasks as modeling the structure of a standard and for recording what parts of a standard are tested by specific tests.

Contact: Alan Goldfine, A266 Technology Bldg., 301/975-3252.
To help protect data communicated through computer networks, NBS researchers are developing protocols for secure key distribution and secure data transmission using public key algorithms. The protocols will protect keys from initia generation to final distribution to all authorized parties. In designing the protocols, researchers will give special attention to preventing substitution of the keys by unauthorized parties. The protocols will also transmit keys and data within a large computer network with automated communications capabilities. And they will be consistent with those used in the Open Systems Interconnection Reference Model. The project results will be the basis for an NBS publication on public key cryptographic protocols.

Contact: Miles E. Smid, A216 Technology Bldg., 301/975-2938.

In the area of global communications, NBS researchers are attempting to define present and future data communication applications; performance metrics; and characteristics of satellite channels, public data networks, and local area networks In addition, they will develop simulation and analytical models to permit performance predictions for open systems protocol end systems and various subnetworks. Through various simulation experiments and analytical calculations, NBS researchers will identify areas worthy of future investigation via live experiments. These live experiments will be performed to verify the significant simulation and analytical results

Contact: John F. Heafner, B217 Technology Bldg., 301/975-3618.

NBS is pursuing the development of several graphics standards and is working on related conformance testing and measurement techniques for graphics software. Specifically, Bureau researchers are testing the implementation of the graphical kernel system (GKS), the computer graphics metafile (CGM), GKS for three dimensions, and the programmers hierarchical interactive graphics standard (PHIGS) for conformance to the existing and emerging federal, national, and international standards. The researchers are attempting to

\section{Public Key Cryptographic Protocols}

Global Communications

Computer Graphics Testing 
derive a general methodology for designing conformance tests, to use this methodology to generate test suites, and to evaluate the test suites for effectiveness. In these studies, they are using the computer graphics laboratory, which contains various computer graphics hardware and computer graphics software systems designed to support standard specifications. An existing test suite for GKS is now available with a FORTRAN interface. Priority is being given to testing methodologies and test suites for PHIGS, CGM, and the conversion of FORTRAN tests for all computer graphics standards to other languages (C, PASCAL, and ADA).

Contact: Mark W. Skall, A265 Technology Bldg., 301/975-3264.

Software National and international standards Engineering groups are working on standards designed to reduce software engineering problems. As part of this effort, the Bureau has been working on specifications for documents representation. The principal specifications are the office document architecture (ODA) and the standard generalized markup language (SGML). NBS researchers are also striving to adopt a Federal Information Processing Standard (FIPS) for UNIXbased operating system environments. In this project, work is centered in three major areas: development of the technical description of the standard; development of guidance for federal agencies on implementation of the standard; and identification of test methods for testing conformance to the standard.

In addition, NBS is evaluating emerging software engineering workstation technology, specifically the use of stand-alone and interconnected workstations for requirements analysis and design, prototyping, verification, and testing.

Contact: Allen L. Hankinson, B266 Technology Bldg., 301/975-3289.

SCSI Verification Standards development is just the first step toward compatibility of computer products. Test and measurement methods are essential for ensuring that products and systems meet the increasingly complex standards. At NBS researchers are setting up a laboratory in which procedures for verifying the conformance of computer storage peripherals to the Small Computer System Interface (SCSI) standard, X3.131-1986, can be developed. The laboratory is being equipped with a variety of SCSI diagnostic tools and peripherals.

Verification of the conformance of storage peripherals to the SCSI standard is difficult because of the wide range of functionality, features, and options included in the standard. This research will result in a procedure for testing and verifying the conformance of storage peripherals to the standard, which can be published and used by others to test storage peripherals.

Contact: William E. Burr, A216 Technology Bldg., 301/975-2914.

The Bureau is designing tools for editing, compiling, and interpreting computer communications protocol specifications. The goal of this research is to advance the state of the art in the application of tools to automatically realize executable implementation of computer communications protocols which are based upon and comply with formal specifications of protocols. As part of this project, Bureau researchers are developing a syntaxdirected editor for Estelle and, using the same grammar, devising a portable compiler for Estelle and the supporting runtime libraries.

Contact: John F. Heafner, B217 Technology Bldg., 301/975-3618.

To enable computer vendors and users to test their computer systems to make sure they conform to networking standards, NBS has established a laboratory for conducting local area network research and development activities. In this specially designed laboratory. researchers are studying conformance testing, performance measurement. analytic and simulation modeling, integration testing and network management. and control of local area networks. Local area networking technology is necessary

\section{Automated Protocol Methods}

Local Area Networking 
for data communication in offices, factories, and laboratory environments where distributed computer network applications are needed. While the interoperability requirements of such networks can lead to unique and expensive communication solutions, the use of standards to achieve product compatibility permits selection of "off-the-shelf" equipment based on cost/performance considerations.

Contact: John F. Heafner, B217 Technology Bldg., 301/975-3618.

Network Security To protect computer systems and Architecture networks, NBS is developing a comprehensive security architecture consistent with the Open Systems Interconnection (OSI) Reference Model. Cryptographic functions are being implemented in certain OSI layers to provide data secrecy, data integrity, and peer entity authentication. The research will combine the security standards for individual OSI layers into a unified security framework. As part of this project, Bureau researchers will define a common interface for cryptographic algorithms and develop a key management methodology capable of providing keys to the cryptographic functions of any layer.

Contact: Miles E. Smid, A216 Technology Bldg., 301/975-2938.

\section{.}

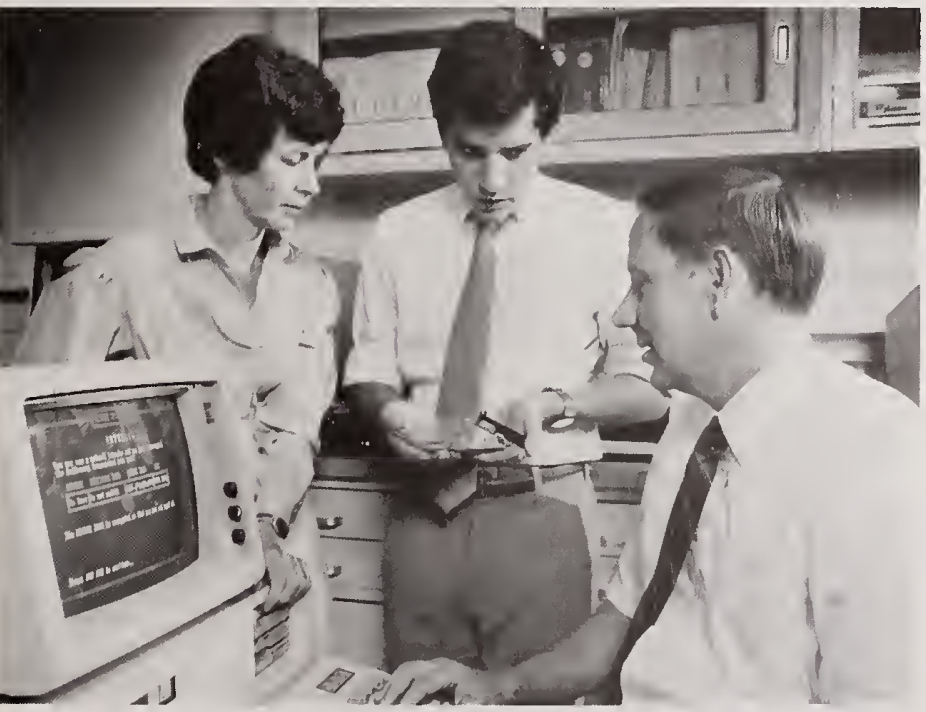

Mathematicians Elaine Barker and Miles Smid (seated) with computer scientist David Balenson discuss cryptographic techniques for securing network communications.
As part of an NBS program aimed at meeting the metrological needs involved in improving signal acquisition and processing systems, Bureau researchers are developing theory, methods, and standards for waveform metrology of conducted signals. In one area, they are conducting the theoretical and experimental research necessary to develop standards for determining the performance of waveform recorders operating nominally below $10 \mathrm{MHz}$. Techniques for synthesis of precision waveforms and for characterization of those waveforms will be developed. Theoretical studies will be conducted on Fourier analysis, deconvolution techniques, and time-domain analysis. Experimental work is planned in precision pulse generation, static and dynamic testing, and assembly and interpretive level programming for hardware control.

Contact: Barry A. Bell, B162 Metrology Bldg., 301/975-2402

Research in the Bureau's antenna metrology program results in reliable techniques and standards for measuring key performance parameters of antennas and components used with satellites, Earth terminals, radars, and communications systems. Scientists are now using near-field scanning to characterize microwave and millimeter-wave antennas. The principal emphasis of this work is on developing spherical scanning to accommodate the largest antennas and adapting near-field scanning to anechoic chamber environments, to permit greatly increased use of near-field scanning in industry.

Contact: Allen C. Newell, Div. 723.05, NBS, Boulder, CO 80303, 303/497-3743.

Research to improve the metrology used to characterize compound semiconductors, especially gallium arsenide, is under way at NBS. Scientists are studying the electrical, optical, and physical properties of gallium arsenide to better understand and characterize presently available device material. They are using a number of measurement techniques in this work, including optical

\section{Waveform \\ Recorder \\ Standards}

Antenna

Measurements

Gallium Arsenide Studies 
absorption, photoluminescence, infrared and Raman spectroscopy, Hall effect and resistivity measurements, capacitancevoltage profiling, deep-level transient spectroscopy, photoconductivity and photocapacitance, infrared imaging, and $x$-ray topography and rocking curve analysis

Contact: Michael I. Bell, 301/975-2081 or Richard A. Forman, 301/975-2047, A305 Technology Bldg.

Integrated Circuit Theoretical and experimental research on

Process semiconductor materials and process

Metrology physics at the Bureau is focused on improving understanding of integrated circuit fabrication technology and increasing capability to measure and characterize fabrication materials and processes. Utilizing processing equipment and techniques appropriate to VLSI chip fabrication, NBS scientists conduct experimental studies on issues related to state-of-the-art semiconductor processing in a well-equipped clean-room semiconductor processing laboratory. For example, researchers are studying ionimplantation damage, oxidation growth and interface trap formation, materials effects caused by ion etching, thin-film properties, and plasma and ion-beam chemistry. Other areas of interest include submicron (submicrometer) photolithog raphy, reactive ion etching, beam processing, and chemical and physical vapor deposition.

Contact: Stanley Ruthberg, 301/9752082 or Gary P. Carver, 301/975-2091, A331 Technology Bldg.

Silicon NBS conducts research in semiconductor Characterization materials, devices, and integrated circuits to provide the necessary basis for understanding measurement-related requirements in semiconductor technology. As part of this program, NBS scientists are employing electrical, optical, and $x$-ray methods to study the resistivity, dopant distribution, and concentration of electrically inactive impurities, such as carbon and oxygen, in silicon. They are developing new or improved techniques for two- and three-dimensional mapping of these properties, refining the quantitative aspects of existing methods, and

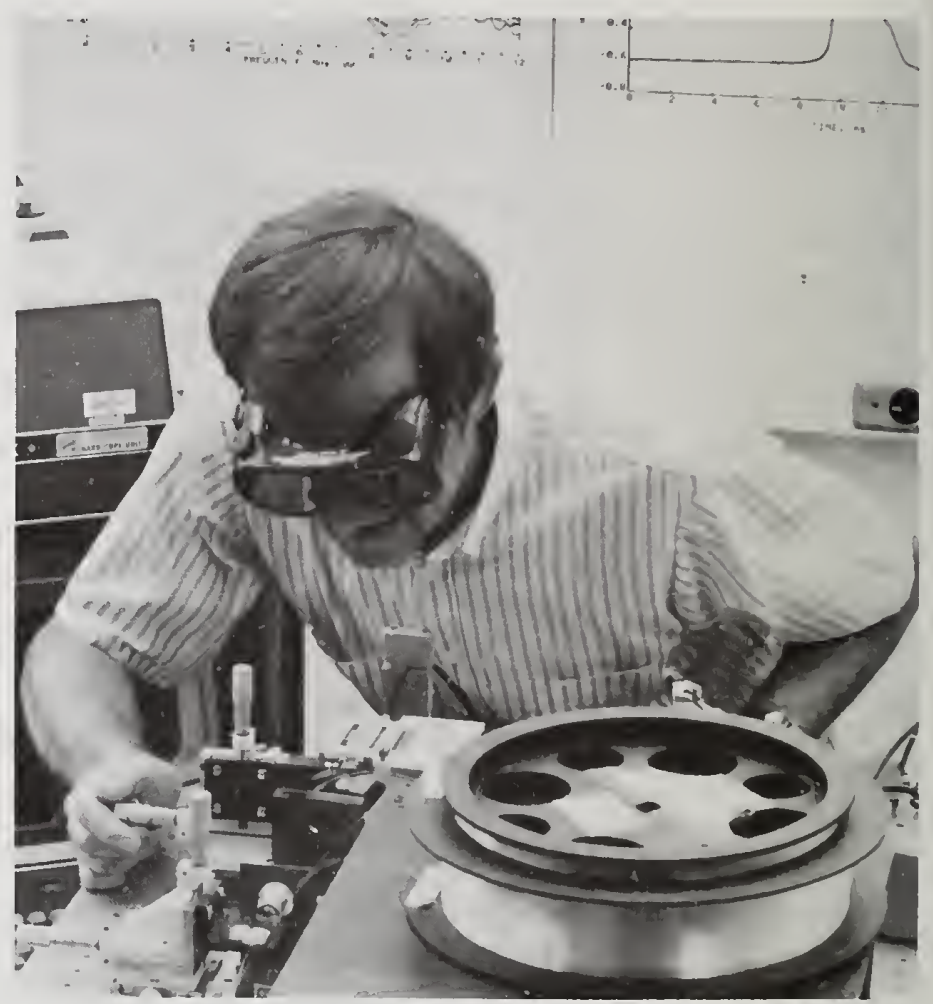

Electrical engineer Robert Gallawa measures the bandwidth of multi-mode optical fibers as part of an NBS program to develop measurement methods that can be used in fiber development, fiber evaluation in the marketplace, and fiber compatibility in communications systems.

developing methods that are nondestructive. Measurements performed for this research include four-probe, spreading resistance, and capacitance-voltage profiling; Fourier transform infrared spectroscopy; deep-level transient spectroscopy; and $\mathrm{x}$-ray topography.

Contact: Michael I. Bell, 301/975-2081 or Jeremiah R. Lowney, 301/975-2048, A305 Technology Bldg.

NBS scientists are developing a basic understanding of magnetic properties of materials and structures to provide a sound basis for measurement techniques. Specifically, researchers are studying precision cryogenic vibrating sample magnetometers, systems based on superconducting quantum interference devices (SQUID's), and laser-based magneto-optical systems. The primary emphasis of this new program is in the
Magnetics 
field of computer-related magnetics, but work is also under way on the basic physics of magnetic materials, such as spin glasses, and on the measurement of very low-level magnetic effects. In a related program in eddy current nondestructive evaluation (NDE), NBS researchers are developing methods for mapping the very small ac magnetic fields associated with eddy current test probes.

Contact: Frederick R. Fickett, 303/497. 3785 or Ronald B. Goldfarb, 303/497. 3650, Div. 724.05, NBS, Boulder, CO 80303.

Cryoelectronics Advances in digital electronics for telecommunications, radar, weapons testing, fusion research, and data processing will demand higher measurement speed than is now possible. The Bureau is now conducting research aimed at exploiting superconducting systems for measurements of extraordinarily high speed, accuracy, and sensitivity. For example, researchers are studying a series array Josephson voltage standard, which is an exciting new approach to simpler, more accurate, and less expensive voltage calibrations and ultra-high-speed analog-to-digital converters and counters, employing as active elements, superconducting quantum interference devices (SQUID's). Their goal is to develop the theoretical and experimental basis for new designs and to fabricate and test these remarkable devices.

Contact: Richard E. Harris, Div. 724.03, NBS, Boulder, CO 80303, 303/497-3776.

Semiconductor To develop physically sound techniques Devices for characterizing, analyzing, and predicting the operation and performance of semiconductor devices, NBS is designing and improving measurement methods for determining critical device parameters for both VLSI-scale and power devices. Research in device modeling includes two-dimensional silicon MOSFET and GaAs MESFET model development and investigations into the validity of the physical assumptions typically employed in silicon bipolar and GaAs device models. Theoretical research is carried out on the transport of ions and electrons in semiconductors for improved process modeling while experimental research on the nature and characterization of electronic states in oxides and at oxide/semiconductor interfaces is under way. Bureau scientists are developing methods for physical and electrical measurements of device and material parameters which are critical for verifying the accuracy and validity of device models. In addition, they are researching the electrical and thermal properties of power semiconductor devices.

Contact: David L. Blackburn, B310 Technology Bldg., 301/975-2053.

Integrated circuit test structures developed by NBS are widely used by the semiconductor industry and other government agencies. These specially designed semiconductor devices can be used to characterize integrated circuit fabrication processes, to evaluate the effectiveness of semiconductor processing equipment, to obtain crucial parameters for device and process models, and for product acceptance. NBS work involves test structure design, mathematical modeling, fabrication, data acquisition, and data analysis. Bureau scientists are conducting research on test structures for random fault detection, yield analysis, and dynamic circuit characterization.

Contact: Loren W. Linholm, B360 Technology Bldg., 301/975-2052.

As part of an NBS program to develop theory, methods, and physical standards for the measurement of electrical quantities in advanced high-voltage/high-power systems, Bureau researchers are developing electro-optical methods for measuring electrical quantities and phenomena. Finite-element code for electric field computation and computer-aided data acquisition and analysis are the focus of theoretical studies. High-voltage ac, dc, and impulse measurements; highspeed cameras; optical multichannel analyzers; and lasers and detectors are addressed in the experimental work.

Contact: Robert E. Hebner, B344 Metrology Bldg., 301/975-2403.

\section{Integrated Circuit Test Structures}


AC Voltage NBS is conducting both theoretical and Standards experimental research on the synthesis of precision ac waveforms for use in ac voltage standards operating nominally below $10 \mathrm{MHz}$. The theoretical work includes Walsh functions and Fourier analysis, time-domain analysis, and precision RMS-to-dc conversion techniques. Experimental work involves high-speed, high-accuracy digital-to-analog conversion; precision, high-speed switching; assembly and interpretive level programming for hardware control; and wideband, fast-settling amplifiers.

Contact: Barry A. Bell, B162 Metrology Bldg., 301/975-2402.

Testing New strategies are needed for evaluating Electronic the performance of complex electronic Systems circuits with the fewest possible tests. NBS is planning a new program which will include theoretical studies of modeling for nonlinear systems, optimization techniques using matrices, statistical and random processes, and artificial intelligence. In addition, experimental work would address test strategies for component and instrument testing; fault diagnosis, function testing, and calibration; and computer analysis using both desktop and supercomputers.

Contact: Barry A. Bell, B162 Metrology Bldg., 301/975-2402.

Raman Scattering Some of the world's most advanced measurement techniques for the rapid advancement of electrical and electronic technology are available at NBS. For example, a Raman spectroscopy facility is used for light scattering studies of liquids, solids, and gases. Specific research being done at the facility includes chemical and structural analysis of thin films and ion-implanted layers, investigations of impurities and other defects in semiconductors, studies of the structural and electrical properties of semiconductor superlattices and quantum wells, and development of new quantitative spectroscopic methods.

In this laboratory, equipment is available for studies of the pressure and temperature dependence of Raman spectra in the range of 4 to $600 \mathrm{~K}$ and up to approximately 100 kbar using a diamond-anvil pressure cell. The labora-

tory is equipped with a Raman microscope for examining regions several micrometers in size, automated digital data acquisition and processing, variable wavelength lasers, and multiple-grating monochromators.

Contact: Michael I. Bell, A305 Technology Bldg., 301/975-2081

NBS scientists are developing measurement methods for characterizing gaseous

Gaseous Dielectrics dielectrics for high-voltage power systems. In this study, the theoretical work is expected to address Boltzmann equilibrium statistics, chemical kinetics code, and computer-aided data acquisition and analysis. Experimental work will focus on high-voltage ac and dc tests, gas chromatograph and mass spectrometer techniques for chemical characterization, and partial discharge measurements. Much of the Bureau's work in advanced power metrology is performed in newly completed highvoltage and high-current laboratories. These facilities can generate voltage pulses with peak amplitudes up to 600,000 volts and current pulses with peak amplitudes up to 100,000 amperes

Contact: Robert E. Hebner, B344 Metrology Bldg., 301/975-2403.

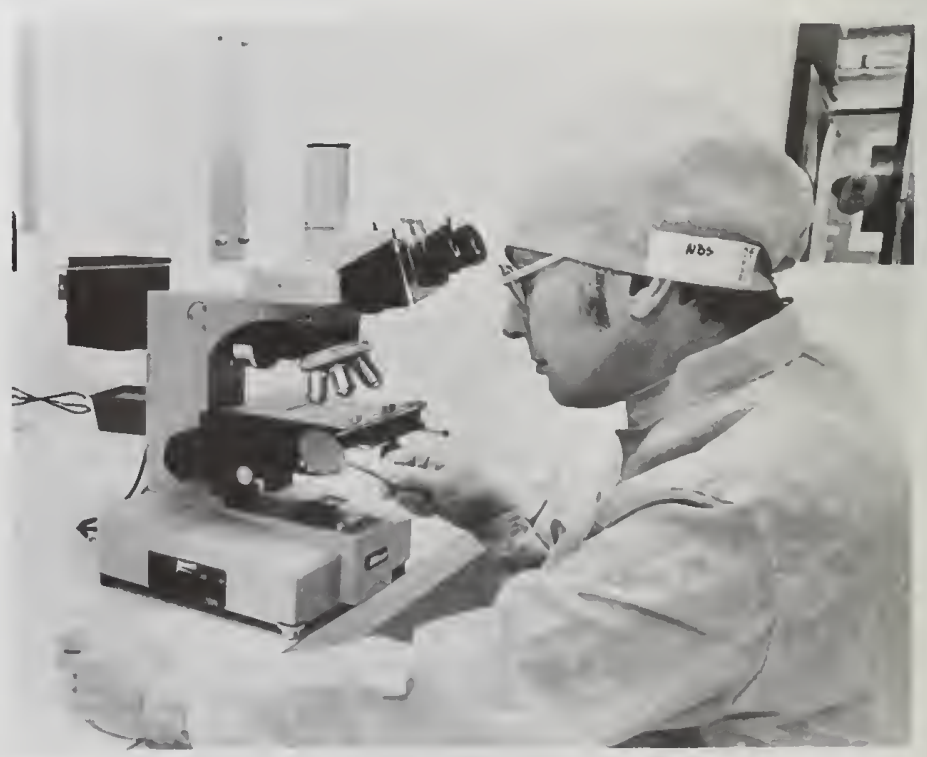

Physicist Gary Carver prepares to inspect a silicon wafer under a microscope in the Bureau's semiconductor processing laboratory. 


\section{Fire Research}

Flammability and Bureau researchers are creating ways to Toxicity measure and characterize the combusti-

Measurement bility of furnishings and building materials and the extent combustion products affect living organisms. Typically NBS research focuses on assessing the appropriate applications of the NBS toxicity screening test, evaluating the extent to which a limited number of gases determine the lethality of combustion products, and developing a methodology for predicting soot and gas production in full-scale fires from benchscale methods. In addition, Bureau researchers are generating a detailed model for the burning of upholstered furniture, devising a measurement methodology to predict the propensity of cigarettes to ignite soft furnishings, and aiding the use of the cone calorimeter in engineering and code applications

Contact: Vytenis Babrauskas, A363 Polymer Bldg., 301/975-6679.

Fire Simulation Over the past decade, NBS researchers have developed many computer models of various aspects of fire. Research in this project is aimed at providing the expedient transfer of scientifically based technology from NBS to the professional user community and creates a link between the computer-based activity of the Bureau and others doing similar or complementary work. Researchers develop engineering systems for design application as well as expert systems, collect supporting data and programs, and operate a working and training laboratory dedicated to computer modeling and other fire safety computations.

Contact: Harold E. Nelson, A247 Polymer Bldg., 301/975-6869.

Hazard Analysis The United States has one of the worst fire records in the industrialized world. The Bureau is providing the scientific and engineering bases needed by manufacturers and the fire protection community to reduce both these losses and the cost of fire protection. One project involves the development of predictive, analytical methods which permit the quantitative assessment of hazard and risk from fires. The Bureau bases these methods on numerical modeling, but also includes hand calculation methods for estimating hazards and production of design curves/tables to be used by architects and engineers. To ensure widespread use, the necessary data must be readily available, and data input and presentation must be in terms readily understandable by the average professional. Thus, the projects include a strong emphasis on state-of-the-art computer graphics and computer-aided design techniques.

Contact: Richard W. Bukowski, B356 Polymer Bldg., 301/975-6879.

Predicting fire growth requires a fundamental understanding of elemental fire processes. Therefore, the Bureau is attempting to develop predictive methods to describe the processes of fire growth and elemental processes of fire growth and extinction as related to gaseous, liquid, and solid fuel combustion and the performance of fire safety systems. Researchers are probing fire growth within a single compartment, the spread of fire to adjoining spaces in multicompartment structures, and the action of fire suppressants. In addition, they are examining the overall dynamics of the fire scenario and related elemental processes such as ignition, flame spread, pyrolysis, extinction, and fire-related transport processes. For these studies, they utilize mathematical techniques, experimental studies, correlations, and similitude methods to develop predictions.

Contact: James G. Quintiere, B250 Polymer Bldg., 301/975-6863.

NBS is conducting research to develop a science base for the gasification and combustion of natural and synthetic polymers, particularly for the more applied fire research activities. For example, NBS scientists are working on the kinetics and heats of combustion for wood, theoretical modeling of thermal degradation of polymers, smoldering research, and detailed degradation mechanisms of polymers.

Contact: Takashi Kashiwagi, B258 Polymer Bldg., 301/975-6693.
Fire Growth and Extinction

\section{Polymer Combustion Research}




\section{Fracture and Deformation}

Welding The integrity of welded joints is a primary Research concern in the design and fabrication of engineering structures. Therefore, NBS is directing research toward improving the fracture resistance of weldments and assessing weld quality by nondestructive evaluation (NDE) techniques. Specific goals of the research are to improve the fracture toughness in the heat-affected zone of steel weldments and in the weld metal of materials for low-temperature service and to better weld soundness through increased understanding of the metal transfer process in arc welding. In addition, Bureau researchers will develop electromagnetic acoustic transducers (EMAT's) for ultrasonic inspection of weldments. The research is conducted in the Bureau's welding and NDE laboratories which house equipment for shielded metal arc, gas metal arc, and gas tungsten arc welding; radiographic, ultrasonic, eddy-current, and penetrant techniques for NDE of weldments; acoustoelastic techniques for residual stress measurements; and a precision power supply, high-speed photography, and laser shadow techniques for metal transfer studies. Recently, NBS scientists developed techniques for porosity reduction in aluminum weldments, spatter reduction in gas metal arc welding of steels, flaw size measurements in steel welds using ultrasonics, and residual stress measurements in aluminum weldments.

Contact: Thomas A. Siewert, 303/497. 3523 or A. Van Clark, 303/497-3159, Div. 430, NBS, Boulder, CO 80303.
To ensure the safety and reliability of engineering structures, the Bureau is working to establish a sound technical basis for codes and standards for structures. Specifically, researchers plan to develop a rational basis for material toughness requirements, allowable stress levels, minimum service temperatures, and weld quality standards. NBS capabilities include test equipment with load capacities up to $6,000,000$ pound-force, numerical analysis of cracked structures, experimental mechanics, and fractographic techniques. Bureau scientists evaluate materials performance at cryogenic and elevated temperatures, under static and dynamic loading, in corrosive and hydrogen-charging environments, and with random amplitude loading. They have recently completed a fitness-forservice evaluation of steam boilers, a failure analysis of a refinery pressure vessel, dynamic crack arrest tests on structural steels, and a new test method for measuring the fracture toughness of weldments.

Contact: Harry I. McHenry, Div. 430, NBS, Boulder, C0 80303, 303/497-3268.
Fracture Mechanics 


\section{Manufacturing Engineering}

Automated Automated manufacturing systems

Manufacturing include flexible manufacturing systems

Systems and machine tools into which intelligent

Hardware sensors and controls of the production processes, including the machining itself, have been embedded. To support such developments by industry, NBS conducts several research programs. In manufacturing systems, scientists are investigating generic control systems, full-task programming, data organization, sensor organization, system initialization, and the object-data analogy. In the area of small displacement measurement, researchers are developing transducers with $100-\mathrm{kHz}$ bandwidths for measuring fractional micro-inch displacements over wide temperature ranges for the next generation coordinate measuring machine.

Their efforts in real-time error correction of machine tools are centered on extending microprocessor-based techniques for real-time correction of the kinematic (as well as static) machine errors and tool wear. They are studying transient waves in solids by investigating the origin, propagation, measurement, and direct-and inverse-prediction of micro-mechanical waves in acoustic emission and ultrasonics. Furthermore, Bureau researchers are focusing their studies of transducer fields on the fields produced in solids by electromechanical transducers by modeling and measuring of the fields point-by-point within the solids.

Contact: Donald S. Blomquist, B108 Sound Bldg., 301/975-6600.

Precision The precision-engineering industries

Engineering create and use precision mechanical machines for production applications, such as high-tolerance diamond-turned optical disks as information storage media. NBS conducts research in the design and high-accuracy performance of several types of next-generation machines. Bureau scientists are focusing their studies of thermal modeling of machine tools and coordinate measuring machines (CMM's) on thermal distortions of the geometry of machine tool/CMM's using experimentally-verified finite-element analysis. In robot metrology, researchers are investigating techniques for measuring robot end-effector positioning, including a spherical-system generalization of the extensible ball-bar and a sixtransducer laser-tracking approach. In the area of absolute air refractivity, researchers are developing a prototype refractometer based on a heterodyne tracking relative to a stabilized twofrequency laser. Research on advanced coordinate measuring machines is aimed at devising a sub-0.1 nanometer accuracy coordinate measuring machine based on precision mechanical design, high-resolution interferometry, and electron probe techniques.

Contact: Robert J. Hocken, A109 Metrology Bldg., 301/975-3464.

For those industries which create and use computer-controlled manufacturing systems, software systems are the critical element. NBS provides research for software in automated manufacturing systems in several areas. In automated process planning, researchers are investigating data structures for automated process planning, distributed system architectures, machinable-feature identification, and expert systems/machinelearning for manufacturing systems. Researchers are also studying architectures, algorithms, and techniques for realtime adaptive control, scheduling, and optimization of cell- and workstation-level operations for FMS cell and workstation control systems. In addition, for manufacturing systems architectures, they are devising functional models of manufacturing organizations to standard system architectures.

Contact: Howard M. Bloom, A127 Metrology Bldg., 301/975-3509.

\section{Automated Manufacturing Systems Software}


Robotics The industries which create and apply robotics systems, i.e., intelligent machines which can autonomously operate on the physical environment, range from the makers of industrial robots to the producers of machine vision and other advanced sensory systems. NBS is conducting research on the design of generically applicable control systems for such devices. One project involves image processing architectures. Here, researchers are investigating methods for machine-processing of complex sensory input data employing low-level image processing systems such as PIPE interfaced to high-level symbol-manipulating computer algorithms. Another study concerns world modeling, in which research focuses on methods of constructing computationally efficient world-model representations of threedimensional object descriptions and geometry of visual images to support, for example, spatial reasoning and predictions of dynamic properties of objects.

In addition, Bureau scientists are studying artificial intelligence for robots by developing techniques to bridge the gap between high-level intelligent planning, decision-making, optimization and goal selection, and low-level servo control. In the area of off-line programming, they are studying group technology, interactive graphics, and object-oriented programming as techniques to overcome the limitations of explicit programming required for robot control.

Contact: James S. Albus, B124 Metrology Bldg., 301/975-3418.
In many industrial operations, the wear of Wear of Metals machine components and materials is one of the principal processes that limits the service life of equipment. At NBS an extensive research program on the effects of wear and erosion on materials is under way to investigate many of these industrial problems. NBS scientists are studying a wide variety of wear modes, including adhesive wear, abrasive wear, erosion, galling wear, and lubricated wear. One area of emphasis concerns the effects of materials parameters such as toughness, hardness, ductility, workhardening characteristics, alloy composition, and microstructure.

As part of this program, NBS scientists are also working with industrial researchers to study the galling wear of alloys used in valves and sliding seals. In this research, scientists are using such characterization techniques as scanning and transmission electron microscopy and various surface analytical methods. Research has shown that high-resolution microscopy can frequently be used to identify the causes of wear, for example, through examination of wear debris particles. NBS has many instruments for conducting wear and erosion experiments under controlled conditions and researchers incorporate standard test methods and equipment where appropriate.

Contact: A. William Ruff, B106 Materials Bldg., 301/975-6010.

The use of artificial joints and fracture fixation devices has increased at a rapid rate in recent years. This is due largely to the impressive success of early designs such as the artificial hip joint that used available stainless steel and polyethylene materials. However, as newer types of implants are used for longer times in patients, it has become clear that improved materials are needed for stronger, lighter, more compatible implants. Bureau scientists are studying the mechanical properties and environmental reactions of metals with strong potential for use as implants. They are placing particular emphasis on fatigue
Synthetic Implant Metals 
life, wear, fretting, processing methods, and surface reactions that occur when the materials are placed in the body. In addition, NBS is characterizing the biocompatibility of these materials by observing cellular growth on metallic surfaces and by studying metal/organic reactions. For this project, NBS researchers are working with several manufacturers in the artificial implant industry to examine the properties and performance of relevant materials. The equipment available for this research includes mechanical testing machines, friction and wear testers, scanning and transmission electron microscopes, and electrochemical apparatus.

Contact: Anna C. Fraker, B128 Materials Bldg., 301/975-6009.

Micromechanical The success of many technically Properties of advanced materials, such as composite Materials materials, electromagnetic recording tapes and disks, and integrated circuits, depends on their mechanical performance. These materials have refined, complex, and heterogeneous microstructures, but problems arise when they behave as systems and reflect the overall mechanical contributions of components a micrometer or less in size. Generally, hardness numbers from the indentation resistances of pointed tools are used to determine mechanical performance. However, NBS researchers are developing a new and significantly better solution, which is derived from the hardness testing practice. We have expanded this technique with the NBS dynamic microindentation instrument, an apparatus that can be used to determine mechanical performance within sample volumes of less than a micrometer on a side.

Three important innovations have resulted from this project. Two have been completed: indentations can now be described by continuous measurements of applied loadings and penetration depths, and indentations can be made using loading times ranging from hours down to milliseconds. The Bureau's goal, at present, is to obtain conventional uniaxial tensile properties (Young's Modulus, yield stress, and strain) rather than to use hardness numbers. From an industrial viewpoint, the technique aids development of new materials, studies of thermal and mechanical processing effects, and nondestructive examinations of fracture resistance.

Contact: Robert S. Polvani, B112 Materials Bldg., 301/975-6003.

Corrosion is a major limiting factor in the service life and cost of many products, plants, and equipment. The costs of metallic corrosion to the U.S. economy are estimated to be $\$ 167$ billion in 1985 dollars, $\$ 25$ billion of which could be saved by using existing technology. The National Association of Corrosion Engineers (NACE) and NBS have joined together in a collaborative NACE/NBS Corrosion Data Program to reduce these enormous costs of corrosion. The central focus of the Corrosion Data Program is to establish an evaluated corrosion database that researchers can access easily by computer to obtain data in a number of possible graphical or tabular formats. The researchers are developing evaluation methodologies and a prototype database for areas covering thermodynamic and kinetic corrosion data. NBS scientists are emphasizing interactions with industry both to help set priorities for data projects and as a source of corrosion data for the database.

Contact: Gilbert M. Ugiansky, B254 Materials Bldg., 301/975-6022.

Corrosion has been recognized as one of the major causes of degradation of all kinds of metallic structures, with financial costs well in the billion-dollar range. Since corrosion reactions are of electrochemical nature, electrochemical measurements can give important information about the chemical processes causing the corrosion and the rate of corrosion as well as its forms, whether uniform or localized corrosion. These measurements involve the determination

\section{Corrosion Data \\ Program}

\section{Corrosion Measurements}


of current and potential and their variations with time; the frequency spectrum of the ac impedance of corroding electrodes; and the detection and analysis of the random fluctuations of electrical parameters.

Electrochemical methods for measuring corrosion rates offer the possibility of following the course of the process in a nondestructive way and assessing the effect of various environmental variables. Other electrochemical techniques can shed light on the kinetics of the corrosion reactions and on the critical factors which may contribute either to catastrophic failures or corrosion prevention. For example, Bureau researchers are now using these methods to investigate the electrochemical processes associated with stress corrosion cracking of brass and copper in ammonia solutions and to examine film breakdown and pit initiation on passive metals. Bldg., 301/975-6017.

Process Control Sensors
Contact: Ugo Bertocci, B254 Materials

The rapid solidification of alloys can produce new types of materials unobtainable by conventional means. Because of their special properties, rapidly solidified alloys are being widely investigated and are beginning to be introduced into commercial devices. Equipment is available at the Bureau to study alloys produced by each of the three major kinds of rapid solidification techniques: atomization techniques in which fine liquid droplets are formed and rapidly frozen to produce alloy powder; ribbon techniques in which a very thin liquid stream or ribbon is rapidly solidified; and rapid surface-melting and refreezing techniques in which a directed energy source, such as an electron beam, is scanned across a surface. The emphasis in the NBS work is on measurement and predictive modeling of the rapid solidification processes. Researchers are studying crystalline, metallic glass, and the new quasicrystal alloys recently discovered at NBS. Efforts are under way to apply advanced sensors to an inert gas atomization system to allow feedback and control of this process. Because processing conditions influence alloy homogeneity, microstructure, extended solid solubility, and the production of new alloy phases, control of these features is critical for producing special properties in these new materials.

Contact: John R. Manning, A153

Materials Bldg., 301/975-6157.

noncontact ultrasonic facilities have been designed featuring high-intensity pulsed lasers, electromagnetic acoustic transducers, and laser interferometers. Coupled with state-of-the-art materialsprocessing equipment and expertise. these facilities offer a unique opportunity to ascertain feasibility and develop prototype specifications for a wide spectrum of sensor needs including the measurement of internal temperature, phase transformations, surface-modified layers, porosity, grain size, and inclusion/segregate distributions.

Contact: Haydn N.G. Wadley, A167 Materials Bldg., 301/975-6140.

\section{Rapid \\ Solidification}

.




\section{Neutron Scattering and Diffraction}

has been applied to several bridges in Maryland and will soon be used on bridges in Virginia.

Contact: Edward Escalante, B254 Materials Bldg., 301/975-6014.

Steel Slag The U.S. steel industry needs therThermochemistry mochemical databases and models for the design of new or improved steelmaking processes. To design such processes, NBS initiated programs in three areas. Bureau researchers are measuring refractory and slag thermochemical equilibria, evaluating the scattered literature data, and developing computer models relating experimental data to industrial conditions. NBS has unique experimental facilities for measuring key phase equilibria and kinetics.

Contact: John W. Hastie, A329 Materials Bldg., 301/975-5754.

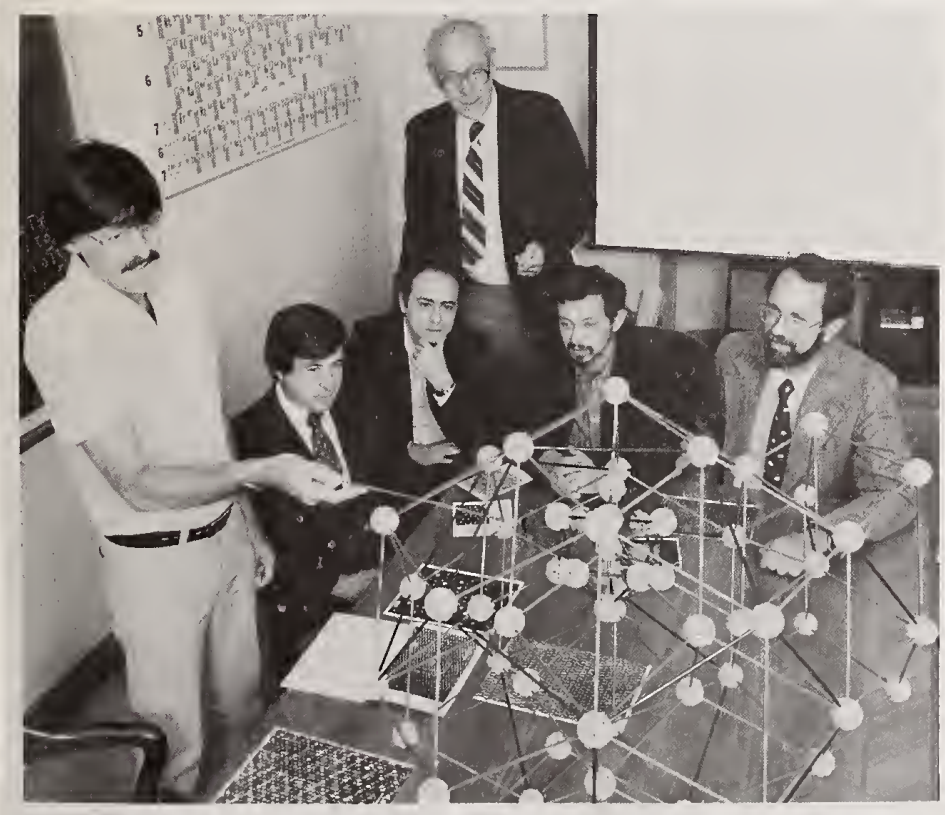

Discussing quasicrystal structures in alloys are (l. to r.) Dan Shechtman, Israel Institute of Technology; Frank Biancaniello, NBS; Denis Gratias, National Science Research Center, France; John Cahn, NBS; Leonid Bendersky, Johns Hopkins University; and Robert Schaefer, NBS. The discovery at NBS of quasicrystal structures in materials challenges a 100-year-old theory of crystallography, and the existence of quasicrystals may make it possible to produce materials with radically different properties.
Precise information on the crystal structure (arrangement of atoms and molecules) in solids is often a key to understanding or improving the properties of modern materials or creating new ones. Many important materials, e.g., ceramics, catalysts, and rapidly solidified alloys, often can be obtained only in powdered form. State-of-the-art capabilities are in place at the Bureau for measuring and analyzing the magnetic structure of polycrystalline materials by neutron diffraction. The facilities available for diffraction experiments for both powders and crystals include a four-circle diffractometer and a multidetector, high-resolution powder diffractometer, as well as instruments which allow measurements in a single-crystal or energydispersive mode.

NBS researchers continue to develop improved methods for accurate structure refinement and for measurement of internal stresses in materials. Currently, a number of industrial scientists are collaborating with NBS staff in neutron diffraction studies of inorganic catalysts, new kinds of ionic conductors for small batteries and fuel cells, improved ceramics for microcircuit substrates and engine components, and highperformance lightweight alloys for advanced aircraft. NBS scientists are also working to determine how residual stress and texture in bulk metals and ceramics affect their application in high-technology products.

Contact: Edward Prince, 301/975-6230 or Antonio Santoro, 301/975-6232, A106 Reactor Bldg.

The vibrational dynamics and diffusion of hydrogen in metals and molecular species in heterogeneous catalysts are studied at the Bureau with inelastic and quasielastic neutron scattering and neutron diffraction. The results of this research reveal the bonding states and atomic scale interactions and diffusion paths in such materials. Recent progress has allowed in-situ spectroscopic studies of hydrogen and molecular species down to 0.1 of an atomic percent. These measurements can provide direct information, for example, on the molecular processes that affect reaction and selective release of chemicals in industrial catalysts and on the local trapping and clustering of hydrogen in metals and semiconductors.
Neutron Diffraction
Neutron Spectroscopy 


\section{Nondestructive Evaluation}

which cause embrittlement of corrosion or changes electronic properties. NBS scientists are working with researchers from several industrial laboratories to study zeolite catalysts. For this research, the equipment available includes threeaxis neutron crystal spectrometers and time-of-flight spectrometers for inelastic scattering along with neutron diffractometers, which measure structural and dynamic processes in the time regime from $10^{-10}$ to $10^{-14} \mathrm{~s}$. Controlled temperature devices are available from 0.3 to $1300 \mathrm{~K}$.

Contact: John M. Rowe, 301/975-6229 or John J. Rush, 301/975-6231, A106 Reactor Bldg.

Small-Angle Small-angle neutron scattering (SANS) is Neutron used to characterize submicron structural Scattering and magnetic properties of materials in the size regime from 1 to $100 \mathrm{~nm}$. The SANS diffraction patterns produced by structural features in this size regime-for example, by small precipitates or cavities in metal alloys, by micropores or cracks in ceramics, by colloidal suspensions and microemulsions, or by polymers and biological macromolecules - can be analyzed to give information on the size and shape of the scattering centers as well as their size distribution, surface area, and number density. This detailed microstructure information is often a key to the prediction or understanding of the performance or failure modes of structural materials and materials processing conditions.

A number of scientists from the chemical, communications, advanced materials, and aerospace industries are already engaged in SANS research at NBS. Their measurements are carried out with long wavelength neutrons on the 8m-long NBS SANS spectrometer which utilizes a 65 by $65 \mathrm{~cm}$ position-sensitive detector to record data over the entire small-angle region simultaneously. Computer-automated apparatus is available for maintaining samples at temperatures from 4 to $700 \mathrm{~K}$ and in magnetic fields up to 20 kilogauss. To extract structural information from the data, the researchers analyze SANS patterns with an interactive color graphics system and related programs.

Contact: Charles J. Glinka, 301/9756242 or James A. Gotaas, 301/975-6243, A106 Reactor Bldg.
Rapidly solidified metal powders provide a basis for alloys which exhibit, after consolidation, high strength-to-weight ratios, improved fracture toughness and fatigue resistance, unique magnetic properties, and high corrosion resistance. Because neither real-time automated process control techniques for producing rapidly solidified metal powders nor techniques for real-time process control of materials exist, NBS recently initiated a model research project which brings together special capabilities that already exist at NBS. Using a pilot facility for studying the automated production of rapidly solidified metal powders, NBS researchers will develop process models, sensors, and adaptive control techniques for producing rapidly solidified metal powders. Two general categories of sensors are involved: one which measures processing parameters, such as temperatures, pressures, and flow rates; and the other includes nondestructive evaluation sensors which measure or monitor non-intrusively important properties or characteristics of the molten, solidifying, or solid metal powders while they are being produced.

Bureau scientists are designing a new, inert gas atomization system, and they are conducting basic research in solidification science to relate the microstructures formed during solidification to the processing conditions and to the subsequent properties. Research at the completed facility is aimed at combining the process models and the real-time sensors with expert computer systems for adaptive control.

Contact: $\mathrm{H}$. Thomas Yolken, B344 Materials Bldg., 301/975-5727.

The eddy-current method of nondestructive evaluation (NDE) is capable of detecting extremely minute cracks, pits, and other discontinuities in surfaces and near-surface regions of metals, alloys, and other electrically conductive materials. In addition, it can be used to characterize near-surface properties of thin coatings, films, and bulk materials. However, to characterize such flaws or properties reliably and reproducibly, eddy-current NDE must be made into a quantitative measurement system. Therefore. the goal of an on-going NBS project
Nondestructive Evaluation for Metal Powder Production 
magnetic resonance spectroscopy is utilized to determine molecular orientation, molecular dynamics, and microstructure on the 1- to $10-\mathrm{nm}$ scale. Microstructural information is deduced from C-13 lineshapes obtained with magic angle spinning or by proton "spin diffusion" experiments in which domainsize information is inferred from the rates at which proton magnetization diffuses in the presence of magnetization gradients. Fourier transform infrared spectroscopy is applied to determinations of molecular architecture, orientation, and molecular processes, such as measurement of the amount of chain scission associated with mechanical deformation of polymers. Optical and electron microscopic studies of polymers elucidate microstructural features, including spherulytic morphologies, lamellar texture, fiber structures, and crystallization habits. To examine the relationships between mechanical performance of polymers and fine structures, NBS scientists are investigating the morphological changes that polymers undergo when they are deformed. Wideand small-angle $x$-ray diffraction techniques, which include the use of position sensitive detectors, are also employed in these investigations.

Contact: Bruno M. Fanconi, A305 Polymer Bldg., 301/975-6770.

Dental and Modern dental and medical materials Medical Materials utilize numerous substances in an array of combinations. NBS has a comprehensive program that is aimed at increasing basic understanding of the causes for failure or poor performance of these materials, proposing and testing new material systems, and transferring the resultant science and technology to industry. The program has the active participation of researchers from the American Dental Association, the National Institutes of Dental Research, dental industries, and universities.

Researchers are working in a number of areas. For example, in a tribology study, scientists are examining wear and degradation of materials in various environments, and in a bioadhesion project, they are making new monomers for adhesion to tissues. In addition, improved resins are being synthesized to produce polymers that have improved properties such as wear resistance, higher strength, resistance to oral fluids, or x-ray opacity.

In an effort to improve the strength of dental systems, different combinations of materials are being designed and tested. They include ceramic-metal, resin matrix composites, metal-cement, cement-tooth, and composite-adhesive-tooth materials. To improve the systems, Weibull statistical analysis is employed to identify the weakest links. Finite element analysis can be applied to define stress states within systems

In the area of precision metal casting processes, researchers are using strain gauges to monitor changes in dimensions of investment patterns at pattern sites. A unique casting monitor has been designed that could also be used for rapidly solidified alloys.

Contact: John A. Tesk, A143 Polymer Bldg., 301/975.6801.

NBS is conducting a number of studies on the electrical properties of polymers. The research is focused on dielectric measurements, fundamentals and applications of piezoelectric and pyroelectric polymers, measurement of space charge distribution within polymer films, and ionic conduction in solid polymers. In the area of dielectric measurements, Bureau scientists have developed instrumentation and data analysis which can measure the dielectric constant and loss of polymer films over a frequency range of $10^{-3}$ to $10^{5} \mathrm{~Hz}$ in less than 30 minutes. This makes it feasible to follow changes in the dielectric spectrum as a function of time, processing conditions, or other parameters.

The toughness, flexibility, low dielectric constant, and an acoustic impedance close to water make piezoelectric polymers ideal for many transducer applications. NBS staff have considerable experience with the fundamental properties of polymers such as polyvinylidene fluoride and its copolymers and can work with industry to develop transducers for novel applications or assist in measuring the properties of new piezoelectric polymers or composites.

Bureau scientists have designed instrumentation and data analysis to measure the charge or polarization distribution across the thickness of polymer
Electrical Properties 
film by analyzing the transient charge response following a pulse of energy on one surface of the film. This technique has been used to detect the presence of non-uniform electric fields in the poling of piezoelectric polymers and is currently being used to investigate the role of space charge in the dielectric breakdown of polymer insulation.

lonic conduction in polymers has potential application in polymeric electrolytes for high-energy density batteries. The dissolution of salts in polymers and their effect on ionic conduction as well as other polymer properties are being studied by a variety of techniques including thermal analysis, $x$-ray diffraction, optical microscopy, and impedance spectroscopy.

Contact: George T. Davis, B320 Polymer Bldg., 301/975-6725

\section{Molecular Thermosets are used extensively in Networks numerous practical applications, espe- cially polymer-based composites. However, knowledge of the basic struc- tural entity of the thermosets, i.e., their molecular network structure, is very limited. Therefore, Bureau researchers have developed a neutron scattering technique that can be used to quantita- tively determine such characteristics of the network as the average distance between crosslinks, the rigidity of the network, the molecular weight distribution between crosslinks, and the topological heterogeneity of the network structure. Current NBS research focuses on developing correlations among chemical compositions, processing conditions, and resulting network structure for epoxy-type polymers. To establish processing- structure-property relations, these materials are then evaluated to determine performance properties such as fracture behavior. The neutron scattering tech- nique is also being used to study how the molecular network deforms when the material is placed under load. These studies can directly benefit industries by providing guidelines for designing and processing polymers for optimum perfor- mance.}

Contact: Wen-li Wu, A209 Polymer Bldg., 301/975-6839.

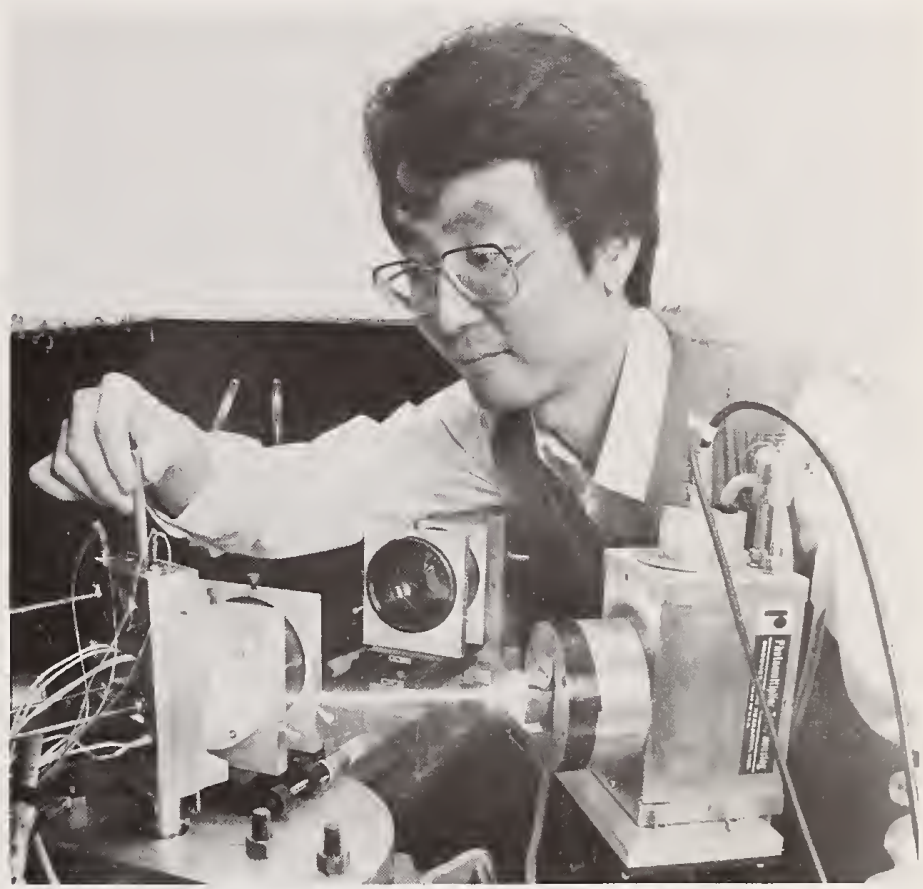

Takahiro Sato, a guest scientist from Osaka University, Japan, uses the temperature-jump light scattering technique to record the phase separation behavior in molecules.

Small-angle $x$-ray scattering (SAXS) can be used to characterize structural elements of polymers, ceramics, and metals in the 1- to $100-\mathrm{nm}$ size range. The Bureau has successfully used this technique to study phase separation. crystallite morphology, molecular dimensions, void formation, pore characteristics, and many others. A state-of-theart, 10-meter SAXS facility has been constructed at NBS for such studies, and it is available for cooperative research. This SAXS facility uses a $12-\mathrm{kW}$ rotating anode source, pinhole collimation, and a two-dimensional position-sensitive detector to provide high resolution and simultaneous recording of all scattering data over the entire small-angle region. In addition, a computer-assisted interactive data reduction and analysis program is available with color graphics display for the two-dimensional image. Structural and dimensional information can be extracted using various available model analysis and curve-fitting procedures.

Contact: Charles C. Han, B208 Polymer Bldg., 301/975-6772.
Small-Angle X-Ray Scattering 
Polymer Blends Blending polymers has become an effective method for producing highperformance engineering polymers. The fundamental data required to design a manufacturing process include the equilibrium phase diagram, the energetic interaction parameter (compatibility) between the blend components, diffusion coefficients, and the interfacial tension. At NBS small-angle neutron scattering has been used to measure the interaction parameter and phase diagram of polymer blends. NBS scientists have developed forced Rayleigh scattering and temperature-jump light scattering techniques to measure polymer-polymer diffusion and other parameters which control phase separation kinetics and morphology.

Currently, Bureau researchers are testing various kinetic theories of phase separation and late-stage coarsening and studying the control of morphology and mechanical properties of microphaseseparated polymer blends. As part of this program, they are working with industry to study the homogenization and phase coarsening of rubber blends.

Contact: Charles C. Han, B208 Polymer Bldg., 301/975-6772.

\footnotetext{
Fluorescence Bureau researchers are evaluating

Monitoring fluorescence spectroscopy, which is used to characterize the structural and dynamical properties of polymer molecules. For example, fluorescence quenching is employed to monitor the uniformity of mixing in polymer blends and in particlefilled polymer melts. In addition, excimer fluorescence is utilized to monitor the cure of thermosetting resins, while fluorescence anisotropy is used to monitor the non-Newtonian flow of polymer solutions and melts. At NBS, the measurements are carried out with a spectrofluorimeter and a nanosecond spectrofluorimeter. In combination with optical fibers, which are used to get the exciting light into the interior of a composite part, the fluorescence techniques are attractive for process monitoring of composites.
}

Contact: Francis W. Wang, B320 Polymer Bldg., 301/975-6725
To help fulfill the needs of laboratories, industry, governmental regulatory agencies, purchasing authorities, and consumers for reliable laboratory testing, NBS operates the National Voluntary Laboratory Accreditation Program (NVLAP). NVLAP is a voluntary system for assessing and evaluating testing laboratories and accrediting those found competent to perform specific test methods or types of tests on products and materials. Through this program, laboratories are accredited for testing a variety of products, including telecommunications equipment, thermal insulation, and radiation dosimeters.

To establish a laboratory accreditation program, NBS works closely with the appropriate industry to develop the technical requirements and documentation needed for the accreditation process. For example, NBS sponsors public workshops to obtain technical guidance from experts in related industry, government, and standards-developing organizations. NBS also works with industry to design proficiency testing requirements, including selection of test methods for which proficiency testing is necessary and appropriate, the frequency of proficiency testing "rounds," and the artifacts to be tested.

Laboratories interested in receiving accreditation are sent a LAP-specific handbook that contains the generic administrative and operational requirements for accreditation as well as the specific technical requirements for accreditation and a set of critical elements which defines those aspects of each test method that an assessor must consider in determining the laboratory's competence. In addition, the laboratory receives application forms and instructions for providing essential information on laboratory ownership, management, key personnel, scope and operation, test method selection, instrumentation and facilities, and other relevant information.

Contact: Harvey W. Berger, A531 Administration Bldg., 3û1/975-4017.

\author{
Laboratory \\ Accreditation \\ Program
}




\section{Radiation Research}

Accelerator The NBS accelerator research program is Research aimed at developing advanced particle accelerators for applications in nuclear physics, free electron lasers, inertial confinement fusion, and dosimetry. The major focus of this NBS program at present is to establish the feasibility and provide the technology needed for $\mathrm{cw}$ accelerators by designing, constructing, and evaluating the performance of a 200$\mathrm{MeV}$ continuous-beam racetrack microtron (RTM) using a room temperature if accelerating section. In addition, NBS scientists are attempting to modify this racetrack microtron for use as a freeelectron laser driver.

To improve industrial applications of particle accelerators, NBS scientists are interested in working with industry researchers to evaluate performance and optimize the RTM design, to design and develop high peak current and high brightness injectors, and design and develop free-electron lasers using the RTM as a driver.

Contact: Samuel Penner, B119 Radiation Physics Bldg., 301/975-5601.

\footnotetext{
Autoxidation/ The kinetic and mechanistic aspects of Antioxidants autoxidation in foods, organic materials systems, and other biochemical systems; the design of appropriate antioxidants for the prevention of the deleterious effects of oxygen; and the extension of the lifetime of chemical and biochemical systems are important to many food, drug, and medical industries. Wideranging NBS research involves investigations in these general areas: the free radical chemistry of organic systems and biochemicals susceptible to autoxidation and free-radical mechanisms and reaction rates of antioxidants, particularly in the case of key components of foodstuffs and other life-supporting substances. Scientists are studying the effects of parameters such as oxygen pressure, temperature, additives, and concentrations on the optimization of these parameters and the development of products less sensitive to oxidation.

NBS researchers are seeking industrial collaboration in the analysis of short- and long-term intermediate species in pulseirradiated oxygenated organic and aqueous solutions; derivation of kinetic data and unraveling of auto- and radiation-stimulated oxidation processes;
}

and design of new antioxidants as protective agents against food spoilage, carcinogenesis, atherosclerosis, and nitrosamine formation in foods. Kinetic and analytical chemical technologies, such as GC/MS, HPLC, spectroscopy, chromatography, electrophoresis, and ESR spectroscopy; high-intensity gammaray sources, pulsed- and continuousbeam electron accelerators; and various organic-chemical analytical equipment are available.

Contact: Michael G. Simic, C206 Radiation Physics Bldg., 301/975-5558

Imaging by use of penetrating radiation is one of the most powerful investigative techniques available to industry for maintaining or improving the quality of products. Designers are now aware of this and are creating components which facilitate such nondestructive testing. Research is under way at NBS that will allow better quantification of the radiographic images. Particularly relevant to image evaluation are computer-based systems that permit pseudo threedimensional images and the implementation of image processing on these or traditional images in real time or near real time.

This research focuses on a number of areas, including image processing for improved imaging of low-contrast or noisy images; adoption of tomographic equipment to industrial needs and measurement of the performance characteristics of such systems; and development of reliable techniques for image storage and retrieval. For these projects, available equipment includes $x$ ray sources, low-energy electron accelerators, and gamma-ray sources as well as state-of-the-art radiologic imaging devices.

Contact: Robert C. Placious, C215 Radiation Physics Bldg., 301/975-5579.

The use of irradiation as a food preservation technique has made research into the chemistry of food processing by ionizing radiations, such as gamma rays and high-energy electrons, important to food industries. NBS is addressing these concerns by studying chemical changes induced by irradiation processing of food

\section{Industrial Radiologic Imaging}

\section{Food Irradiation Chemistry}


components including proteins, fats, sugars, and vitamins. In one project, researchers are selecting radiolytic products which could be used by food processors as indicators to determine whether foods have been irradiated. Other investigations compare thermal and irradiation food processing chemistry and the effect of irradiation processing parameters, such as dose rate and temperature, on food chemistry and quality. Researchers work closely with the Food and Drug Administration and the United States Department of Agriculture to obtain up-to-date information about regulatory and safety requirements for foods and food processing and associated quality control measurements.

Specific areas in which cooperative research is encouraged include kinetic and steady-state analyses of radiolytic products in food components, comparison of energy absorption mechanisms in foods, design of post-irradiation quantitative and qualitative analyses of various foods and their components, and development of food component Standard Reference Materials. Sophisticated analytical techniques such as GC/MS, HPLC, spectroscopy, chromatography, electrophoresis, and ESR spectroscopy as well as high-intensity gamma-ray sources, pulsed-and continuous-beam electron accelerators, and various organic-chemical analytical equipment are available.

Contact: Michael G. Simic, C206 Radiation Physics Bldg., 301/975-5558.

Neutron Fluence NBS researchers are studying industrial Measurement and applications of neutron fluence and dose Neutron Physics determination in the neutron energy region from thermal to $20 \mathrm{MeV}$. They are concentrating on the development of effective methods to transfer personnel protection technology to the private sector. This research provides a basis for standardizing personnel protection control procedures in nuclear reactor and high-energy accelerator operations, for example.

Specific research projects involve: measurement of reference standard neutron reaction cross sections; characterization of reference fission deposits; development of neutron detectors with fast timing; calibrations using standard neutron and gamma-ray fields; and applications of neutron resonance radiography.

Equipment available includes a 100MeV linac-based dedicated pulsed neutron source, a 3-MV pulsed positiveion accelerator, and a 20-MW nuclear reactor.

Contact: Oren A. Wasson, B111 Radiation Physics Bldg., 301/975-5567.

To produce radiation for experimental purposes, NBS has built a synchrotron ultraviolet radiation facility (SURF-II). SURF-II is a $300-\mathrm{MeV}$ electron storage ring that radiates high-intensity synchrotron radiation in the energy range from about $3 \mathrm{~nm}(400 \mathrm{eV})$ in the soft $x$-ray region to wavelengths in the visible region of the spectrum. The radiation is highly collimated, nearly linearly polarized, and of calculable intensity. Six beam lines are available and a user's program is in operation. SJRF-II is well suited for several different types of research, such as atomic, molecular, biomolecular, and solid-state physics; surface and materials science; radiation chemistry and radiation effects on matter; ultraviolet and $\mathrm{x}$-ray optical systems; and the calibration, characterization, and development of spectrometers and detectors.

NBS scientists are particularly interested in conducting collaborative research in: the use of soft $x$-ray synchrotron radiation for high-resolution lithography; measurement of the reflection characteristics of novel, high-efficiency, multilayer far ultraviolet optical devices; and calibration, characterization, and development of spectrometers and detectors.

Other available equipment includes a state-of-the-art multipurpose detector calibration facility, a large ultrahigh vacuum spectrometer calibration chamber, and a beamline dedicated to high-resolution radiation research with a $6.65-\mathrm{m}$ vacuum spectrometer with a resolving power of about 300,000 .

Contact: Robert D. Madden, A249 Physics Bldg., 301/975-3726 or William R. Ott, B206 Metrology Bldg., 301/975-3709

\section{Ultraviolet and \\ Soft X-Ray Radiation Effects}


Optical The U.S. defense, aerospace, health, Pyrometry, lighting, and photographic industries Radiometry, require radiometric measurements for Photometry product development, quality control, and equity in trade. Compatibility of industrial radiometric standards with national and international standards is a key requirement to ensure a competitive position in world markets for these industries. To help ensure reliable radiometric measurements, NBS is conducting radiometric research in the near-ultraviolet, visible, and infrared spectral regions and developing accurate standards and calibration procedures for these regions.

Specific research focuses on calibration of near-infrared pyrometers; geometrically total spectral flux standards; detectorbased radiometric standards; diode-array radiometry, photometry, and colorimetry; low-light-level calibration standards; nearinfrared detectors; infrared spectral radiance standards; and absolute spectral analyzer systems. Available for these projects is sophisticated optical equipment such as tunable dye lasers, electrooptical devices, and interferometers.

Contact: A. Russell Schaefer, A313 Metrology Bldg., 301/975-2331.

Radiation To enhance quality control methods Processing employed in industrial radiation processing of foods and the production and use of medical devices, electronic components, and polymers, NBS researchers are developing standardization and measurement assurance methods related to industrial high-dose applications of ionizing radiation. As part of this program, Bureau scientists are investigating radiation chemical mechanisms and kinetic studies applied to chemical dosimetry systems in the condensed phase, including liquids, gels, thin films, and solid-state detectors. They plan to examine such sensor materials as doped plastics, solid-state matrices, organic dye solutions, semiconductors, scintillators, biopolymers, amino acids, metalloporphyrins, and organic or inorganic aqueous solutions and gels.

In this research, scientists use a number of analytical methods including transmission and fluorescence spectrophotometry, electron spin resonance spectrometry, chemiluminescence photometry, as well as fiber optics analysis, microcalorimetry, pulse radiolysis, and conductivity measurements. Various $x$-ray and gamma-ray sources and electron accelerators with energies in the 0.1 to $10-\mathrm{MeV}$ range are employed. In addition, conventional ultraviolet, visible, and infrared spectrophotometers and spectrofluorimeters, high-intensity gamma-ray sources, pulsed and continuous beam electron accelerators, and various organic-chemical analytical equipment are available.

Contact: William L. McLaughlin, C209 Radiation Physics Bldg., 301/975-5559.

As part of its research to understand and measure various forms of radiation, NBS is conducting vacuum ultraviolet studies involving radiation damage, polymerization of organic molecules, and solar simulation. NBS scientists have worked with iridustry researchers to develop and test vacuum ultraviolet instrumentation, new spectrometer designs, and detector systems, especially for flight in space.

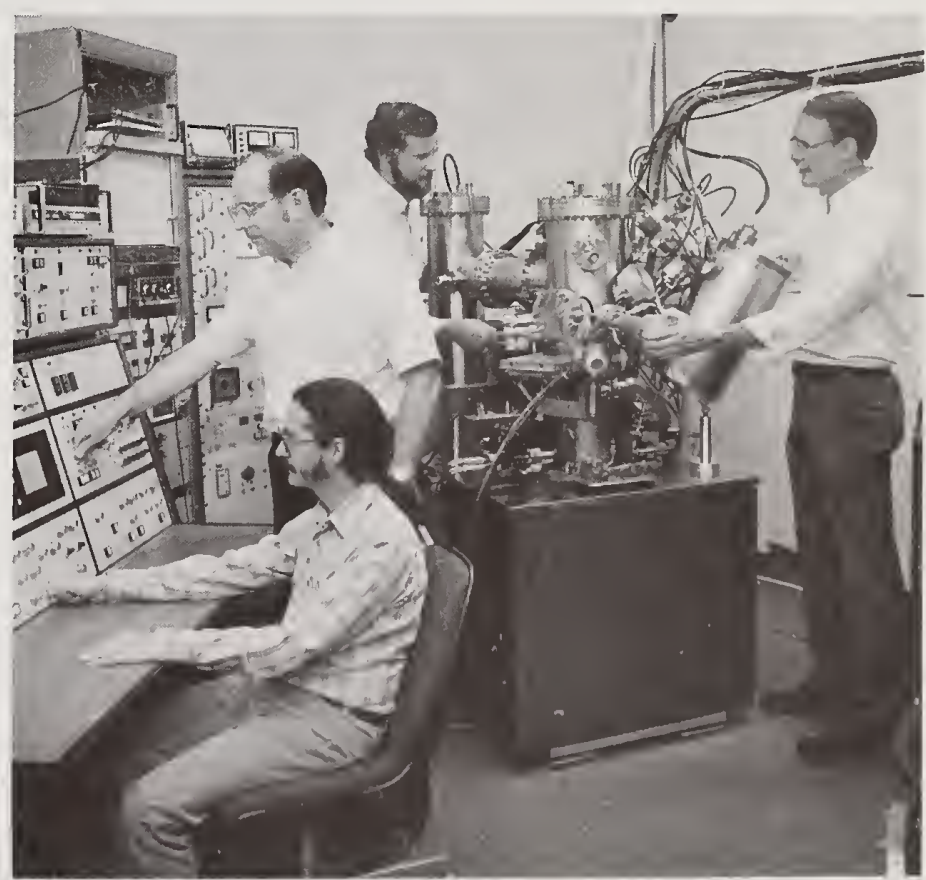

By combining an ultrahigh vacuum, high-resolution scanning electron microscope with a new, compact electron spinpolarization detector, physicists (I. to r.) Gary Hembree, Robert Celotta, John Unguris, and Daniel Pierce can observe simultaneously the magnetic character of a surface and its physical structure over dimensions as small as 100 angstroms. 


\section{Standard Reference Data}

They have also collaborated on special sources, narrow band filters, and lasers.

Bureau scientists are interested in doing cooperative research in several other areas including studying the platinum-neon hollow cathode lamp as a secondary standard, laser plasmas as secondary standards, and a spark discharge light source as a secondary standard. State-of-the-art radiometric facilities and advanced optical equipment are available at the Bureau for these studies.

Contact: J. Mervin Bridges, 301/9753228 or Jules Z. Klose, 301/975-3230, B66 Physics Bldg.

Submicron Well-characterized, focused electron Physics beams are used to interrogate the properties of surfaces and magnetic materials on a microscopic scale. NBS conducts a broad-based research program exploring the physics of submicron systems with electron microprobes. The program includes studies of electron-surface interactions, surface magnetism, electron interaction theory, and electron spin polarization phenomena. Extensive experimental facilities are available for this research, including sources and detectors of spin polarized electrons, a scanning electron microscope equipped for secondary electron spin polarization analysis, and a scanning tunneling microscope

Scanning electron microscopy with polarization analysis (SEMPA) is a unique measurement capability developed by NBS scientists to allow the high-resolution $(\sim 10 \mathrm{~nm})$ imaging of magnetic microstructure independently of surface topography. With this technique, they are studying fundamental magnetic phenomena, the properties of highdensity magnetic recording media, and small-particle magnetism. Bureau researchers are using the scanning tunneling microscope (STM) to study surface topography at the atomic level, mechanisms of film growth at the submonolayer level, reconstructions of semiconductor surfaces, and, ultimately, correlation of surface microstructure with macroscopic properties.

Contact: Robert J. Celotta, B210 Metro logy Bldg., 301/975-3710
Using computers to provide timely and inexpensive access to reliable chemical, physical, and materials data contributes greatly to increased productivity and lower manufacturing costs of industrial operations. The NBS Standard Reference Data program works closely with industry to develop the tools and standards necessary for computerizing industrial technical data-the numbers, graphics, tables, and text that convey research results.

The National Standard Reference Data System, coordinated by NBS, is a major focal point in the United States for the evaluation of chemical, physical, and materials properties data. The NBS Standard Reference Data program has made a commitment to provide national leadership in the computerization of these evaluated data, including database design, data interchange standards, and data dissemination.

NBS research combines expertise in the physical sciences with specialized knowledge in computer science. Areas of activity include preparation of databases of evaluated data and predictive software for use on personal computers, conversion and combination of published data compilations into searchable databases, standardization of formats for scientific information, development of expert systems, and establishment of distributed systems linking scientific databases Computer and telecommunications capabilities at NBS offer challenging alternatives to the handling of these projects, with state-of-the-art mainframes, minis and micros, database management systems, graphics, and applications software available.

Contact: David R. Lide, A323 Physics Bldg., 301/975-2200
Computerization of Standard Reference Data 


\section{National Bureau of Standards}

The National Bureau of Standards was established by an act of Congress on March 3, 1901. The Bureau's overall goal is to strengthen and advance the nation's science and technology and facilitate their effective application for public benefit. To this end, the Bureau conducts research and provides: (1) a basis for the nation's physical measurement system; (2) scientific and technological services for industry and government; (3) a technical basis for equity in trade; and (4) technical services to promote public safety. The Bureau's technical work is performed by the National Measurement Laboratory, the National Engineering Laboratory, the Institute for Computer Sciences and Technology, and the Institute for Materials Science and Engineering

\section{The National Measurement Laboratory}

Provides the national system of physical and chemical measurement; coordinates the system with measurement systems of other nations and furnishes essential services leading to accurate and uniform physical and chemical measurement throughout the nation's scientific community, industry, and commerce; provides advisory and research services to other government agencies; conducts physical and chemical research; develops, produces, and distributes Standard Reference Materials; and provides calibration services. The laboratory consists of the following areas:

- Standard Reference Data

- Measurement Services

- Basic Standards

- Radiation Research

- Chemical Physics

- Analytical Chemistry

\section{The National Engineering Laboratory}

Provides technology and technical services to the public and private sectors to address national needs and to solve national problems; conducts research in engineering and applied science in support of these efforts; builds and maintains competence in the necessary disciplines required to carry out this research and technical service; develops engineering data and measurement capabilities; provides engineering measurement traceability services; develops test methods and proposes engineering standards and code changes; develops and proposes new engineering practices and develops and improves mechanisms to transfer results of its research to the ultimate user. The laboratory consists of the following centers:

- Applied Mathematics

- Electronics and Electrical Engineering

- Manufacturing Engineering

- Building Technology

- Fire Research

- Chemical Engineering

\section{The Institute for Computer Sciences and Technology}

Conducts research and provides scientific and technical services to aid federal agencies in the selection, acquisition. application, and use of computer technology to improve effectiveness and economy in government operations in accordance with Public Law 89-306 (40 U.S.C. 759), relevant Executive Orders, and other directives; carries out this mission by managing the Federal Information Processing Standards Program, developing federal ADP standards guidelines, and managing federal participation in ADP voluntary standardization activities; provides scientific and technological advisory services and assistance to federal agencies; and provides the tech nical foundation for computer-related policies of the federal government. The institute consists of the following centers: - Programming Science and Technology

- Computer Systems Engineering

\section{The Institute for Materials Science and Engineering} Conducts research and provides measurements, data, standards, reference materials, quantitative understanding, and other technical information fundamental to the processing, structure, properties, and performance of materials; addresses the scientific basis for new advanced materials technologies; plans research around cross-country scientific themes such as nondestructive evaluation and phase diagram development; oversees Bureau-wide technical programs in nuclear reactor radiation research and nondestructive evaluation; and broadly disseminates generic technical information resulting from its programs. The institute consists of the following divisions:

- Nondestructive Evaluation

- Inorganic Materials

- Fracture and Deformatıon

- Polymers

- Metallurgy

- Reactor Radiation 
